\title{
A NONCOMMUTATIVE WEIERSTRASS PREPARATION THEOREM AND APPLICATIONS TO IWASAWA THEORY
}

\author{
OTMAR VENJAKOB
}

\begin{abstract}
In this paper and a forthcoming joint one with Y. Hachimori 15 we study Iwasawa modules over an infinite Galois extension $k_{\infty}$ of a number field $k$ whose Galois group $G=G\left(k_{\infty} / k\right)$ is isomorphic to the semidirect product of two copies of the $p$-adic integers $\mathbb{Z}_{p}$. After first analyzing some general algebraic properties of the corresponding Iwasawa algebra, we apply these results to the Galois group $X_{n r}$ of the $p$-Hilbert class field over $k_{\infty}$. The main tool we use is a version of the Weierstrass preparation theorem, which we prove for certain skew power series with coefficients in a not necessarily commutative local ring. One striking result in our work is the discovery of the abundance of faithful torsion $\Lambda(G)$-modules, i.e. non-trivial torsion modules whose global annihilator ideal is zero. Finally we show that the completed group algebra $\mathbb{F}_{p} \llbracket G \rrbracket$ with coefficients in the finite field $\mathbb{F}_{p}$ is a unique factorization domain in the sense of Chatters [8].
\end{abstract}

\section{INTRODUCTION}

One of the highlights of noncommutative Iwasawa theory so far is the structure theory of Iwasawa modules due to Coates, Schneider and Sujatha. In 10 they proved that every torsion $\Lambda(G)$-module decomposes - up to pseudo-isomorphism - into the direct sum of cyclic modules, where $\Lambda(G)=\mathbb{Z}_{p} \llbracket G \rrbracket$ denotes the Iwasawa algebra of a pro- $p$-group $G$ belonging to a certain class of $p$-adic groups, viz the class of $p$-valued ones. In this paper we want to illustrate some features of their theory in the possibly easiest noncommutative example: we take as group the semi-direct product $G=H \rtimes \Gamma$ of two copies of the $p$-adic integers $\mathbb{Z}_{p} \cong H \cong \Gamma$ with a non-trivial action of $\Gamma$ on $H$.

If $M$ is a finitely generated left module over a ring $R$, we define, as usual, $\operatorname{Ann}(M)$ to be the set of all $r$ in $R$ such that $r \cdot M=0$. If $R$ is commutative and $M$ is a torsion $R$-module, we always have $\operatorname{Ann}(M) \neq 0$. However, when $R$ is noncommutative, we define a torsion $R$-module $M$ to be faithful or bounded, according as $\operatorname{Ann}(M)=0$ or $\operatorname{Ann}(M) \neq 0$. When $R=\Lambda(G)$, with $G=H \rtimes \Gamma$ as above, both types of modules occur. But what is very striking is that those modules which occur most naturally in arithmetic ( see below and [15]), namely the modules which are finitely generated over $\Lambda(H)$, are either faithful or pseudo-null. In

Date: 11.03 .02 .

Key words and phrases. Iwasawa algebra, skew power series rings, Weierstrass preparation theorem, faithful modules, $p$-Hilbert class field.

During this research, the author has been supported by the EU Research Training Network "Arithmetical Algebraic Geometry" at the Department of Pure Mathematics and Mathematical Statistics, Cambridge. 
fact, an even stronger result is true. Let $\mathcal{C}$ be the subcategory of all pseudo-null modules in the category $\Lambda(G)$-mod of all finitely generated $\Lambda(G)$-modules.

Theorem (Theorem 6.3). Let $M$ be a $\Lambda(G)$-module which is finitely generated over $\Lambda(H)$. Then he image of $M$ in the quotient category $\Lambda$-mod/C is a completely faithful, cyclic object.

Here an object $\mathcal{M}$ of the quotient category $\Lambda$-mod $/ \mathcal{C}$ is called completely faithful if all its nonzero subquotient objects are faithful (see $\S$ 6).

In section 8 we will consider $p$-adic Lie extensions $k_{\infty}$ over a number field $k$ whose Galois group $G=G\left(k_{\infty} / k\right)$ is isomorphic to a semidirect product of the type studied above. Such examples arise by adjoining the $p$-power roots of an element $\alpha \epsilon k$ which is not a root of unity to the cyclotomic $\mathbb{Z}_{p}$-extension of $k$, assuming that the latter field contains the $p$ th roots of unity $\mu_{p}$, or $\mu_{4}$ if $p=2$. If we take for example $\alpha=p$ and $k=\mathbb{Q}\left(\mu_{p}\right)$, we shall show that the Galois group $X_{n r}$ of the $p$-Hilbert class field gives rise to a completely faithful object.

At this opportunity we should mention a further result on the module $X_{n r}$. The remarkable duality between the inverse and direct limit of the $p$-primary ideal class groups in the tower $k_{\infty}$ of number fields which was observed by Iwasawa for the cyclotomic $\mathbb{Z}_{p}$-extension - compare also with results of McCallum [21] or Nekovar [24] in the case of multiple $\mathbb{Z}_{p}$-extensions - holds in the following (noncommutative) case

Theorem (Theorem 8.2). Let $k=\mathbb{Q}\left(\mu_{p}\right)$ and $k_{\infty}=k\left(\mu_{p^{\infty}}, p^{p^{-\infty}}\right)$. Then, there is an injective homomorphism of $\Lambda(G)$-modules

$$
C l\left(k_{\infty}\right)(p)^{\vee} \rightarrow \mathrm{E}^{1}\left(X_{n r}\right)
$$

with pseudo-null cokernel, i.e. the Pontryagin dual of the direct limit of the p-ideal class groups is pseudo-isomorphic to the Iwasawa-adjoint of the inverse limit. In particular, if $X_{n r}$ is pseudo-null, then the ideal class group $C l\left(k_{\infty}\right)(p)=0$ vanishes.

It is easy to see that $X_{n r}$ is non-zero if and only if $p$ is an irregular prime. We know no example at present where $X_{n r}$ is not pseudo-null, and it may well be that is always the case. In a forthcoming paper 15] (with Y. Hachimori) we will prove that certain Selmer groups or rather their Pontryagin dual are indeed nonzero completely faithful objects in the quotient category. Thus faithful modules definitely occur in the world of arithmetic. Hence, in these cases, one cannot hope to obtain a link with L-functions via a global annihilator or a characteristic ideal, which simply does not exist for such completely faithful modules.

To obtain the algebraic results on modules over $\Lambda(G)$, where now in general $G$ is a uniform pro- $p$-group which is isomorphic to the semidirect product $H \rtimes \Gamma$ of a uniform pro-p-group $H$ with $\Gamma \cong \mathbb{Z}_{p}$, we proceed as follows: Firstly, we identify $\Lambda(G)$ with the skew power series ring $=R[[Y ; \sigma, \delta]]$ over the subalgebra $R:=\Lambda(H)$ of $\Lambda(G)$. Secondly, we study in some generality skew power series rings $A:=R[[Y ; \sigma, \delta]]$ the definition of which is given in section 2 and prove a generalized Division Lemma and Weierstrass Preparation Theorem for such 
rings (see Theorem 3.1 and corollary 3.2). Here we give only one remarkable consequence of this preparation theorem. By $\Lambda(G)-\bmod ^{H}$ and $\mathcal{C}^{H}$ we denote the category of $\Lambda(G)$-modules which are finitely generated as $\Lambda(H)$-module, and its full subcategory of those pseudo-null $\Lambda(G)$-modules, which are finitely generated over $\Lambda(H)$, respectively. The skew polynomial $\operatorname{ring} R[Y ; \sigma ; \delta] \cong R[Z, \sigma], Z=$ $Y+1$, possesses as a ring of quotients $Q[Z ; \sigma]$ where $Q=Q(H)$ denotes the skewfield of fractions of $\Lambda(H)$. We prove the following

Theorem (Theorem 5.8). The quotient category $\Lambda(G)-\bmod ^{H} / \mathcal{C}^{H}$ can be identified with a full subcategory of the category $Q[Z ; \sigma]$-mod of finitely generated $Q[Z ; \sigma]$-modules

$$
\Lambda(G)-\bmod ^{H} / \mathcal{C}^{H} \subseteq Q(H)[Z ; \sigma]-\bmod .
$$

Under this identification, the equivalence classes of simple objects of the quotient category correspond uniquely to equivalence classes of maximal left ideals of $Q[Z ; \sigma]$ which contain a distinguished polynomial. Furthermore, bounded objects correspond to bounded $Q[Z ; \sigma]$-modules.

An analogous statement holds if we replace $Q[Z ; \sigma]$ by the skew Laurent polynomial ring $Q\left[Z, Z^{-1} ; \sigma\right]$. In the case where $G \cong \mathbb{Z}_{p} \rtimes \mathbb{Z}_{p}$ with non-trivial action, an easy calculation shows that the $\operatorname{ring} Q\left[Z, Z^{-1} ; \sigma\right]$ is simple, hence does not admit any bounded module and the first theorem above follows.

For a commutative power series ring in several variables over a complete discrete valuation ring the Weierstrass preparation theorem is one key means of proving the unique factorization property. At least for the completed group algebra (of the semidirect product $G$ ) with coefficients in the finite field $\mathbb{F}_{p}$ a similar result holds which can hopefully be extended to the full Iwasawa algebra with $\mathbb{Z}_{p}$-coefficients:

Theorem (Theorem 7.1). The ring $\mathbb{F}_{p} \llbracket G \rrbracket=\mathbb{F}_{p}[[X, Y ; \sigma, \delta]]$ is an UFD in the sense of Chatters [8].

For the precise definition of a (noncommutative) unique factorization domain (UFD) we refer the reader to section 7 . To the author's knowledge this is the first example of a complete noncommutative ring of global cohomological dimension greater than 1 , which is a UFD.

ACKNowledgements. I would like to thank John Coates most warmly not only for inviting me to DPMMS, Cambridge University, but also for his great interest and helpful comments on this project. Also I am very grateful to Yoshitaka Hachimori for valuable, stimulating discussions and reading parts of the manuscript. Susan Howson is thanked for helpful suggestions. Finally, I thank the EU Research Training Network "Arithmetic Algebraic Geometry" for its support and DPMMS for its hospitality.

Convention. In this paper, the ring $R$ is always associative and with a unit element. When we are talking about properties related to $R$ like being "noetherian", an "ideal", a "unit", of a certain "global dimension", etc. we always mean the left and right property if not otherwise stated. But by an $R$-module we 
usually mean left $R$-module (not a bi-module). By a local ring $R$ we mean a ring in which the non-units form a proper ideal, which is then automatically maximal as left, right and two-sided ideal. Equivalently, $R$ has both a unique left and a unique right maximal ideal, which amounts to the same as the quotient $R / J(R)$ of $R$ by its Jacobson radical being a skewfield.

\section{SKEW POWER SERIES RINGS}

We begin by recalling the definition of skew power series rings, which have already been extensively studied in the literature (see [23], citeli, [11]). Let $R$ be a ring, $\sigma: R \rightarrow R$ a ring endomorphism and $\delta: R \rightarrow R$ a $\sigma$-derivation of $R$, i.e. a group homomorphism satisfying

$$
\delta(r s)=\delta(r) s+\sigma(r) \delta(s) \text { for all } r, s \in R .
$$

Then the (formal) skew power series ring

$$
R[[X ; \sigma, \delta]]
$$

is defined to be the ring whose underlying set consists of the usual formal power series $\sum_{n=0}^{\infty} r_{n} X^{n}$, with $r_{n} \in R$. However, the multiplication of two such power series is based on the right multiplication of $X$ by elements of $R$ which is defined by the formula

$$
X r=\sigma(r) X+\delta(r)
$$

This clearly implies that, for all $n \geq 1$, we have

$$
X^{n} r=\sum_{i=0}^{n}\left(X^{n} r\right)_{i} X^{i}
$$

for certain elements $\left(X^{n} r\right)_{i} \in R$. In other words, we have and in general the multiplication is given by

$$
\left(\sum r_{i} X^{i}\right)\left(\sum s_{j} X^{j}\right)=\sum_{n} c_{n} X^{n}
$$

where

$$
c_{n}=\sum_{j=0}^{n} \sum_{i=n-j}^{\infty} r_{i}\left(X^{i} s_{j}\right)_{n-j} .
$$

To grant that the above series converges to an element $c_{n} \in R$ we have to impose further conditions: Either $\delta=0$ (then $X^{n} r=\sigma^{n}(r) X^{n}$, i.e. the sum $c_{n}=\sum_{i+j=n} r_{j} \sigma^{i}\left(s_{j}\right)$ is finite) or we assume that $R$ is a complete ring with respect to the $I$-adic topology, $I$ some $\sigma$-invariant ideal, and that it holds

$$
\delta(R) \subseteq I, \delta(I) \subseteq I^{2}
$$

This implies by induction that $\delta\left(I^{k}\right) \subseteq\left(I^{k+1}\right)$ for all $k \geq 0\left(I^{0}=R\right.$ by convention $)$ and the following lemma shows that the multiplication law in $R[[X ; \sigma, \delta]]$ is well defined.

Lemma 2.1. (i) If $r \in I^{k}$ then $\left(X^{n} r\right)_{i} \in I^{k+n-i}$ for all $i, n \geq 0$.

(ii) If $\sigma$ is an isomorphism satisfying $\sigma(I)=I$, then for any $n \epsilon \mathbb{N}: r \epsilon I^{k} \backslash I^{k+1}$ if and only if $\left(X^{n} r\right)_{n} \in I^{k} \backslash I^{k+1}$. In particular, $r \neq 0$ if and only if $\left(X^{n} r\right)_{n} \neq 0$. 
Proof. All statements follow by induction, the case $n=1$ following from the defining relation $X r=\sigma(r) X+\delta r$, while the induction step uses

$$
\left(X^{n+1} r\right)_{j}=\sigma\left(\left(X^{n} r\right)_{j-1}\right)+\delta\left(\left(X^{n} r\right)_{j}\right)
$$

and $\left(X^{n} r\right)_{n}=\sigma^{n}(r)$.

Our interest in this kind of rings stems from applications in algebraic number theory where the following example naturally occurs, see section 8 .

Example 2.2. Let $G$ be the semi-direct product $G=\Gamma_{1} \rtimes \Gamma_{2}$ of the pro- $p$ groups $\Gamma_{1}$ and $\Gamma_{2}$, both isomorphic to the additive group of $p$-adic integers $\mathbb{Z}_{p}$, where the action of $\Gamma_{2}$ on $\Gamma_{1}$ is given by a continuous group homomorphism $\rho: \Gamma_{2} \rightarrow \operatorname{Aut}\left(\Gamma_{1}\right) \cong \mathbb{Z}_{p}^{*}$. Then the completed group algebras $\mathbb{Z}_{p} \llbracket G \rrbracket$ and $\mathbb{F}_{p} \llbracket G \rrbracket$ of $G$ with coefficients in $\mathbb{Z}_{p}$ and $\mathbb{F}_{p}$ respectively are isomorphic to the skew power series rings

$$
\begin{aligned}
& \mathbb{Z}_{p} \llbracket G \rrbracket \cong \mathbb{Z}_{p}[[X]][[Y ; \sigma, \delta]]=: \mathbb{Z}_{p}[[X, Y ; \sigma, \delta]] \\
& \mathbb{F}_{p} \llbracket G \rrbracket \cong \mathbb{F}_{p}[[X]][[Y ; \sigma, \delta]]=: \mathbb{F}_{p}[[X, Y ; \sigma, \delta]]
\end{aligned}
$$

where

(i) $X:=\gamma_{1}-1, Y:=\gamma_{2}-1$ for some generators $\gamma_{i}$ of $\Gamma_{i}, i=1,2$.

(ii) Setting $R:=\mathbb{Z}_{p}[[X]]\left(\mathbb{F}_{p}[[X]]\right)$ and $\epsilon:=\rho\left(\gamma_{2}\right)$ the ring automorphism $\sigma$ is induced by $X \mapsto(X+1)^{\epsilon}-1$, while $\delta=\sigma-$ id.

Indeed, conferring Lazard 's work $\llbracket 19 \rrbracket$ (see also $\llbracket 13 \rrbracket)$ the ring $\mathbb{Z}_{p} \llbracket G \rrbracket\left(\mathbb{F}_{p} \llbracket G \rrbracket\right)$ has $X^{i} Y^{j}, 0 \leq i, j<\infty$, as a (complete) $\mathbb{Z}_{p}\left(\mathbb{F}_{p}\right)$-basis and it is straight forward to verify that the relation between $X$ and $Y$ is described via $\sigma$ and $\delta$. Furthermore, $R$ is obviously complete with respect to the topology induced by its maximal ideal $\mathfrak{m}$ which is generated by $X$ and $p$. Since $\sigma$ is induced by choosing another generator of $\Gamma_{1}$ under the isomorphism $\mathbb{Z}_{p}[[X]] \cong \mathbb{Z}_{p} \llbracket \Gamma_{1} \rrbracket$ we see that $\sigma(\mathfrak{m})=\mathfrak{m}$. Thus we only have to verify that $\delta\left(\mathfrak{m}^{k}\right) \subseteq \mathfrak{m}^{k+1}$ for $k=0,1$ : Since $\delta$ and $\sigma$ are $\mathbb{Z}_{p}$ $\left(\mathbb{F}_{p}\right)$ - linear the claim follows immediately from the special case $r=X$ observing also that $\delta\left(\mathbb{Z}_{p}\right)=0$ (and analogously for $\left.\mathbb{F}_{p}\right)$ :

$$
\begin{aligned}
\delta X & =\sigma(X)-X \\
& =(X+1)^{\epsilon}-1-X \\
& =\sum_{i \geq 1}\left(\begin{array}{c}
\epsilon \\
i
\end{array}\right) X^{i}-X \\
& =(\epsilon-1) X^{k}+\text { terms of higher degrees. }
\end{aligned}
$$

Since $p \mid(\epsilon-1)$ we conclude $\delta(X) \subseteq \mathfrak{m}^{2}$.

Example 2.3. The previous example generalizes immediately to the following situation: Let $H$ be a uniform pro- $p$ group on which the group $\Gamma \cong \mathbb{Z}_{p}$ acts via a continuous group homomorphism $\rho: \Gamma \rightarrow \operatorname{Aut}(H)$ and assume that the image of $\rho$ is contained in $\Gamma\left(H^{p}\right):=\left\{\gamma \in \operatorname{Aut}(H) \mid[\gamma, H] \subseteq H^{p}\right\}$ where $[\gamma, h]=h^{\gamma} h^{-1}$ for 
$h \epsilon H$. Then it is easy to see that the semidirect product $G=H \rtimes \Gamma$ is again an uniform pro- $p$ group. Its Iwasawa algebras $\Lambda(G):=\mathbb{Z}_{p} \llbracket G \rrbracket$ and $\Omega(G):=\mathbb{F}_{p} \llbracket G \rrbracket$ of $G$ with coefficients in $\mathbb{Z}_{p}$ and $\mathbb{F}_{p}$ are isomorphic to the skew power series rings over the Iwasawa algebras $\Lambda(H)$ and $\Omega(H)$, respectively,

$$
\begin{aligned}
& \Lambda(G) \cong \Lambda(H)[[Y ; \sigma, \delta]], \\
& \Omega(G) \cong \Omega(H)[[Y ; \sigma, \delta]],
\end{aligned}
$$

where $Y:=\gamma-1$ for some generators $\gamma$ of $\Gamma$, the ring automorphism $\sigma$ is induced by $h \mapsto h^{\gamma}$ and again $\delta=\sigma-$ id. Indeed, since for any $h \epsilon H$

$$
\begin{aligned}
\delta(h-1) & =\sigma(h-1)-(h-1) \\
& =h^{\gamma}-h \\
& =\left(g^{p}-1\right) h(\text { for some } g \in H \text { by assumption) } \\
& \epsilon \mathfrak{m}^{2} \text { (see the claim in the proof of [28, lemma 3.24]), }
\end{aligned}
$$

$\delta(\mathfrak{m}) \epsilon \mathfrak{m}^{2}$ and the conditions on $\sigma$ and $\delta$ are fulfilled as above.

We call a uniform pro-p-group $G$ polycyclic if it contains a finite chain of subgroups

$$
1=G_{0} \subseteq G_{1} \subseteq \cdots G_{i} \subseteq G_{i+1} \subseteq \cdots G_{n}=G
$$

such that $G_{i}$ is normal in $G_{i+1}$ with quotient $G_{i+1} / G_{i} \cong \mathbb{Z}_{p}$ for $0 \leq i<n$. Then, as $\mathrm{S}$. Howson suggests, one can identify $\Lambda(G)$ with the skew power series ring in $n$-variables

$$
\Lambda(G) \cong \mathbb{Z}_{p}\left[\left[X_{0}, \ldots, X_{n-1} ; \sigma_{1}, \delta_{1}, \ldots, \sigma_{n}, \delta_{n}\right]\right]
$$

which is defined recursively by

$$
\begin{aligned}
\Lambda\left(G_{1}\right) & =\mathbb{Z}_{p}\left[\left[X_{0}\right]\right], \\
\Lambda\left(G_{i+1}\right) & =\Lambda\left(G_{i}\right)\left[\left[X_{i} ; \sigma_{i}, \delta_{i}\right]\right],
\end{aligned}
$$

where $\sigma_{i}$ and $\delta_{i}$ are defined as above.

Example 2.4. A rather trivial special case of the previous example is $R[[X]]$ where $R$ is any (noncommutative) ring, $\sigma=\mathrm{id}$ and $\delta=0$. In the Iwasawa theory of elliptic curves there occur naturally open subgroups $G \subseteq G L_{2}\left(\mathbb{Z}_{p}\right)$ such that $G=H \times \Gamma$ where $H=S L_{2}\left(\mathbb{Z}_{p}\right) \cap G$ and $\Gamma \cong \mathbb{Z}_{p}$ is the center of $G$. Then the Iwasawa algebra $\Lambda(G)$ can be identified with the power series ring $\Lambda(H)[[T]]$ in one (commuting) variable with coefficients in the sub Iwasawa algebra $\Lambda(H)$.

In case $\delta=0$ the following properties of $A=R[[X ; \sigma]]$ reflect the corresponding ones of $R$ ([23, thm. 1.4.5, thm. 7.5.3], [20, Chap. II Cor. 3.2.12, Chap. III Thm. 3.4.6]):

Proposition 2.5. Let $A=R[[X ; \sigma]]$. Then the following holds:

(i) If $\sigma$ is injective and $R$ is an integral domain, then $A$ is an integral domain.

(ii) If $\sigma$ is injective and $R$ a division ring, then $A$ is a principal left ideal domain (Note that in this case $\delta=0$ because of the above conditions). 
(iii) If $\sigma$ is an automorphism and $R$ is left (or right) Noetherian, then $A$ is left (respectively right) Noetherian. In this case, the left (or right) global dimensions of $A$ and $R$ are related by

$$
\mathrm{gl} A=\mathrm{gl} R+1
$$

(iv) If $\sigma$ is an automorphism and $R$ is a maximal Noetherian order, then $A$ is a maximal Noetherian order.

(v) If $\sigma$ is an automorphism and $R$ an Auslander regular ring, then $A$ is an Auslander regular ring.

In order to prove similar results in the general case we first have to introduce some filtrations on $A=R[[X ; \sigma, \delta]]$. We assume for the rest of this section that $R$ is a local ring with maximal ideal $\mathfrak{m}$ which is complete and separated with respect to its $\mathfrak{m}$-adic topology. Then we set $F_{0} A:=A$ and define, for $n \geq 1, F_{n} A$ to be the set of all $f=\sum_{k=0}^{\infty} c_{k} X^{k}$ such that $c_{k} \in \mathfrak{m}^{n}$ for all $k \geq 0$. Finally, we set

$$
G_{0}:=A, \quad G_{n}:=\sum_{i=0}^{n}\left(F_{n-i} A\right) X^{i}, \quad n \geq 1,
$$

where the sum is a sum of left By definition, both filtrations $G_{i}$ and $F_{i}$ are exhaustive and $F_{i} A \subseteq F_{j} A$ respectively $G_{i} A \subseteq G_{j} A$ holds for all $i \geq j$. The following lemma shows that they are indeed filtrations of rings:

Lemma 2.6. For all $n, m \geq 0$, it holds: $F_{n} A \cdot F_{m} A \subseteq F_{n+m} A$ and $G_{n} A \cdot G_{m} A \subseteq$ $G_{n+m} A$.

Proof. The assertion for the filtration $F_{i}$ follows immediately from the formula 2.2, lemma 2.1 and by the fact that $\mathfrak{m}^{k}$ is closed with respect to the $\mathfrak{m}$-adic topology for all $k$ (see [20, Ch. I 3.1(c)]). Concerning the second filtration it suffices by distributivity to prove that

$$
\left(F_{n-i} A\right) X^{i}\left(F_{m-j} A\right) X^{j} \subseteq \sum_{k=0}^{n+m}\left(F_{n+m-k} A\right) X^{k}
$$

for all $0 \leq i \leq n$ and $0 \leq j \leq m$ holds. Using the multiplicativity of $F_{i}$ just proved this will follow immediately once we have shown the relation

$$
X^{i}\left(F_{k} A\right) \subseteq \sum_{j=0}^{i}\left(F_{k+i-j} A\right) X^{j} .
$$

So let $f=\sum f_{i} X^{i}$ in $F_{k} A$. The defining relation 2.1 implies that $X f$ decomposes into the sum

$$
X f=\sum_{i=0}^{\infty} \delta\left(f_{i}\right) X^{i}+\left(\sum_{i=1}^{\infty} \sigma\left(f_{i-1}\right) X^{i-1}\right) X,
$$

the first summand of which lies in $F_{k+1} A$ while the second one belongs to $\left(F_{k} A\right) X$ by lemma 2.1. The proof is accomplished by induction. 
Note that the two-sided ideals $G_{k} A$ (apply the lemma with $n$ or $m$ equal to 0 ) can be described as follows:

$$
G_{k} A=\left(\prod_{i=k}^{\infty} R X^{i}\right) \times \mathfrak{m} X^{k-1} \times \cdots \times \mathfrak{m}^{k} X^{0}=\prod_{i=0}^{\infty} \mathfrak{m}^{k-i} X^{i}
$$

for all $k \geq 0$, where $\mathfrak{m}^{l}:=R$ for negative integers $l$ and where $A$ is identified with $A=\prod_{i=0}^{\infty} R X^{i}$.

Lemma 2.7. The topology on $A$ induced by the filtration $G_{i} A$ coincides with the product topology of $A \cong \prod_{i=0}^{\infty} R$, where $R$ is endowed with its $\mathfrak{m}$-adic topology.

Proof. With respect to the product topology a neighbourhood basis of $0 \in A$ consists of

$$
A_{k}:=\prod_{i=0}^{k-1} \mathfrak{m}^{k} X^{i} \times \prod_{i=k}^{\infty} R X^{i}, k \geq 0 .
$$

It is easy to see that the $B_{k}$ and $G_{i}$ are cofinal to each other and hence induce the same topology on $A$.

Since the product of complete Hausdorff topological spaces is again a complete Hausdorff topological space we obtain

Corollary 2.8. The ring $A$ is a complete Hausdorff topological ring with respect to its product topology respectively the topology which is induced by the filtration $G_{i}$.

Proposition 2.9. There is a canonical isomorphism of graded rings

$$
g r_{G} A \cong\left(g r_{\mathfrak{m}} R\right)[\bar{X} ; \bar{\sigma}]
$$

where the latter ring bears the mixed grading with respect to the grading of $g r_{\mathfrak{m}} R$ and the " $\bar{X}$-adic" grading. Here $\bar{X}$ means the image of $X$ and $\bar{\sigma}$ is induced by $\sigma$.

Proof. The above description 2.3 implies immediately that

$$
G_{k} / G_{k+1} \cong \prod_{i=0}^{k} \mathfrak{m}^{i} / \mathfrak{m}^{i+1} \bar{X}^{k-i}
$$

holds. Thus

$$
\begin{aligned}
\bigoplus_{k=0}^{\infty} G_{k} / G_{k+1} & \cong \bigoplus_{i, l \geq 0} \mathfrak{m}^{i} / \mathfrak{m}^{i+1} \bar{X}^{l} \\
& \cong \bigoplus_{i \geq 0} g r_{\mathfrak{m}} R \bar{X}^{i} \\
& \cong\left(g r_{\mathfrak{m}} R\right)[\bar{X} ; \bar{\sigma}]
\end{aligned}
$$

As a corollary it turns out that $A=R[[X ; \sigma, \delta]]$ reflects the properties of $g r_{\mathfrak{m}} R$ :

Corollary 2.10. If $g r_{\mathfrak{m}} R$ is 
(i) integral and $\bar{\sigma}$ is injective, then $A$ is integral.

(ii) left (or right) Noetherian and $\bar{\sigma}$ is an automorphism, then $A$ is left (respectively right) Noetherian. If, in addition, the left (or right) global dimension of $g r_{\mathfrak{m}} R$ is finite, then so is the left (respectively right) global dimension of $A$ and it holds

$$
\mathrm{gl} A \leq \mathrm{glg} r_{\mathfrak{m}} R+1
$$

(iii) a Noetherian maximal order, $R$ complete and $\bar{\sigma}$ is an automorphism, then $A$ is a maximal Noetherian order.

(iv) Noetherian, $R$ complete and $\bar{\sigma}$ is an automorphism, then $A$ is a Zariski-ring.

(v) Auslander regular, $R$ complete and $\bar{\sigma}$ is an automorphism, then $A$ is Auslander regular.

Proof. Assuming conditions (i) or (ii), [23, thm. 1.2.9] tells that $g r_{G} A \cong\left(g r_{\mathfrak{m}} R\right)[\bar{X} ; \bar{\sigma}]$ is integral, respectively Noetherian. This implies the same properties for the ring $A$ by [23, thm 1.6.7, thm. 1.6.9]. The statement concerning the global dimensions follow similarly by [23, thm. 7.5.3 (iii), cor. 7.6.18]. Now let us assume (iii). Then $g r_{G} A \cong\left(g r_{\mathfrak{m}} R\right)[\bar{X} ; \bar{\sigma}]$ is a maximal order according to [20, Chap. II Cor. 3.2.12], thus $A$ is a maximal order by [10, lem 2.6]. Under the condition (iv) $A$ is complete with respect to the filtration $G_{i}$. Since $g r_{G} A$ is Noetherian, $A$ is a Zariski ring by [20, Chap. II thm. 2.1.2]. To prove (v) it suffices to show that $g r_{G} A$ is Auslander regular ([20, Chap. III thm. 2.2.5]), which is contained in [20, Chap. III thm. 3.4.6 (1)].

We close this section proving that under our current conditions on $R$ the skew power series ring $A$ is a local ring.

Proposition 2.11. An element $f=\sum f_{i} X^{i} \in A$ is a unit (in $A$ ) if and only if the constant term $f_{0}$ is a unit in $R$. In particular, $A$ is a local ring.

Proof. The "only if" part follows by considering the canonical surjective map $A \rightarrow$ $A / G_{1} A \cong R / \mathfrak{m}$. Now assume without loss of generality that $f_{0}=1$ (otherwise replace $f$ by $f_{0}^{-1} f$ ) and set $h:=f_{0}-f \in G_{1} A$. Then, due to completeness of $A$ with respect to $G_{i} A$, the "geometric series" $\sum_{i>0} h^{i}$ converges to an element $g \in A$, which is a left and right inverse of $f=1-h$. That means that the non-units consist precisely of the elements of $\mathfrak{M}=G_{1} A$.

\section{The Weierstrass preparation theOrEm}

Let $R$ be a (not necessarily commutative) local ring with maximal (left) ideal $\mathfrak{m}$ and suppose that $R$ is separated and complete with respect to its $\mathfrak{m}$-adic topology. As usual we denote the residue class skewfield $R / \mathfrak{m}$ by $k$. As before we assume that $\sigma: R \rightarrow R$ is a ring-automorphism satisfying $\sigma(\mathfrak{m})=\mathfrak{m}$, i.e. $\sigma$ induces also a ring automorphism $\sigma: k \rightarrow k$, while $\delta: R \rightarrow R$ is a $\sigma$-derivation such that $\delta(R) \subseteq \mathfrak{m}, \delta(\mathfrak{m}) \subseteq \mathfrak{m}^{2}$. In particular, $\delta$ operates trivially on $k$. Therefore there is a canonical surjective reduction map: 


$$
{ }^{-}: R[[X ; \sigma, \delta]] \rightarrow k[[X ; \sigma]] .
$$

In example $2.2 \sigma$ will turn out to be the identity on $k$. For any $f=\sum a_{i} X^{i} \in A:=$ $R[[X ; \sigma, \delta]]$ the reduced order $\operatorname{ord}^{\text {red }}(f)$ of $f$ is defined to be the order of the reduced power series $\bar{f}$, i.e.

$$
\operatorname{ord}^{r e d}(f)=\min \left\{i \mid a_{i} \in R^{*}\right\} .
$$

Theorem 3.1. Let $f \epsilon A$ be a power series with finite reduced order $s:=\operatorname{ord}^{\text {red }}(f)<$ $\infty$. Then $A$ is the direct sum

$$
A=A f \oplus \bigoplus_{i=0}^{s-1} R X^{i}
$$

of the $R$-modules $N:=$ Af and $M:=\bigoplus_{i=0}^{s-1} R X^{i}$.

Proof. (compare to Bourbaki's proof in the commutative case [3, §3 no.8]) We first prove that $N \cap M=0$ :

For any element of this intersection one has an equality

$$
\begin{aligned}
r_{0}+r_{1} X+\ldots+r_{s-1} X^{s-1} & =\left(\sum b_{j} X^{j}\right) f \\
& =\sum c_{n} X^{n} .
\end{aligned}
$$

Comparing the coefficients we conclude that for $k \geq 0$

$$
\begin{aligned}
0 \stackrel{!}{=} c_{s+k}= & \sum_{0 \leq j \leq s+k} b_{i}\left(X^{i} a_{j}\right)_{s+k-j} \\
= & b_{k}\left(X^{k} a_{s}\right)_{k}+\sum_{0 \leq j \leq s+k} b_{i}\left(X^{i} a_{j}\right)_{s+k-j} . \\
& (i, j) \neq(k, s)
\end{aligned}
$$

To prove that $b_{j}=0, j \geq 0$, it suffices to see that $b_{k} \in \mathfrak{m}^{n}$ for all $k$ and $n$. By double induction, let us assume that $b_{i} \in \mathfrak{m}^{n-1}$ for all $i$ and $b_{i} \in \mathfrak{m}^{n}$ for $i<k$. Since $a_{s} \in R^{*}$, so is $\left(X^{k} a_{s}\right)_{k}$ by lemma 2.1 and the following claim implies that $b_{k} \in \mathfrak{m}^{n}$.

Claim: $b_{i}\left(X^{i} a_{j}\right)_{s+k-j} \in \mathfrak{m}^{n}$ for all $0 \leq j \leq s+k,(i, j) \neq(k, s)$.

For $i<k$ the claim is obvious because of $b_{i} \epsilon \mathfrak{m}^{n}$ then. So let us assume $i \geq$ $k,(i, j) \neq(k, s)$. We have to deal with three cases separately:

(i) If $i+j \leq s+k$ then $\left(X^{i} a_{j}\right)_{s+k-j}=0$.

(ii) If $i+j=s+k$ then $j=s+k-i<s$ (note that $i=k$ would imply that $j=s$ ), i.e. $a_{j} \in \mathfrak{m}$ by the definition of $s$ and the claim follows from lemma 2.1 . 
(iii) If $i+j>s+k$ then $i-(s+k-j) \geq 1$, i.e. the claim follows from the same lemma.

We still have to prove that $N+M=A$. Since $a_{s} \in R^{*}$ and $A$ is local we can write

$$
f=\sum_{i=0}^{s-1} a_{i} X^{i}+g X^{s}
$$

with $g \in A^{*}$. Setting $G:=g^{-1}$ and

$$
h:=-G\left(\sum_{i=0}^{s-1} a_{i} X^{i}\right)=-G\left(f-g X^{s}\right)=X^{s}-G f
$$

we claim that all coefficients of $h$ are in $\mathfrak{m}$. Indeed,

$$
h_{n}=\sum_{j \leq \min \{n, s-1\}} G_{i}\left(X^{i} a_{j}\right)_{n-j} \in \mathfrak{m}
$$

using lemma 2.1 because $a_{j} \in \mathfrak{m}$ for $j<s$.

Now let $r$ be an arbitrary element in $A$. Recursively we can find a series $q^{(n)}=$ $\sum_{l=0}^{\infty} q_{l}^{(n)} X^{l}, n \in \mathbb{N}$, of elements in $A$ such that

$$
q^{(0)} X^{s} \equiv r \bmod M
$$

and

$$
q^{(n+1)} X^{s} \equiv q^{(n)} h \bmod M \text { for } n \geq 0
$$

(Just cut off the terms of degrees less than $s$ with respect to $X$ and then factor out $X^{s}$ on the right). Since $q_{l}^{(n+1)}=\left(q^{(n)} h\right)_{s+l}$ it is easily seen that $q_{l}^{(n+1)} \in \mathfrak{m}^{n+1}$ for all $l$ using the fact that the coefficients of $h$ lie all in $\mathfrak{m}$. Hence, due to the completeness of $R, \sum q^{(n)}$ converges to an element $q \epsilon A$. The relations

$$
\left(q^{(0)}+\cdots+q^{(n)}\right) X^{s} \equiv r+\left(q^{(0)}+\cdots+q^{(n-1)}\right) h \bmod M
$$

imply that

$$
\begin{aligned}
r & \equiv q\left(X^{s}-h\right) \bmod M \\
& =(q G) f \epsilon A f .
\end{aligned}
$$

In analogy with the commutative situation we call a monic polynomial $F=$ $X^{s}+a_{s-1}+\cdots+a_{1} X+a_{0} \in R[X ; \sigma, \delta] \subseteq R[[X ; \sigma, \delta]]$ distinguished or Weierstrass polynomial (of degree $s$ ) if $a_{i} \in \mathfrak{m}$ for all $0 \leq i \leq s-i$. Here, $R[X ; \sigma, \delta]$ denotes the skew polynomial ring in one variable over $R$ with respect to $\sigma$ and $\delta$.

Corollary 3.2. Under the assumptions of the theorem $f$ can be expressed uniquely as the product of an unit $\epsilon$ of $A$ and a distinguished polynomial $F \in A$ :

$$
f=\epsilon F \text {. }
$$


Proof. According to the theorem the element $X^{s} \in A$ can be uniquely written as

$$
X^{s}=v f-H
$$

where $H=\sum_{i=0}^{s-1} g_{i} X^{i}$ and $v \in A$. Reducing the coefficients mod $\mathfrak{m}$ we obtain

$$
X^{s}=\bar{v} \bar{f}-\sum_{i=0}^{s-1} \bar{g}_{i} X^{i} .
$$

Since $\operatorname{ord}(\bar{f})=\operatorname{ord}^{r e d}(f)=s$, i.e. $\operatorname{ord}(\bar{v} \bar{f})=\operatorname{ord}(\bar{v})+\operatorname{ord}(\bar{f}) \geq s$, the coefficients $\bar{g}_{i}$ must all vanish. Therefore

$$
F:=X^{s}+H
$$

is a distinguished polynomial and

$$
X^{s}=\bar{v} \bar{f}
$$

i.e. $\operatorname{ord}(\bar{v})=\operatorname{ord}\left(X^{s}\right)-\operatorname{ord}(\bar{f})=0$. Hence, $v$ is a unit in $A$ and $f=\epsilon F$ with $\epsilon:=v^{-1}$.

Independently, D. Burns and C. Greither 12 have proved another generalized version of the Weierstrass preparation theorem for power series rings in one commuting variable over noncommutative (not necessarily local) rings.

Remark 3.3. Let $J$ be a left ideal of $A=R[[X ; \sigma, \delta]]$. Then $A / J$ is finitely generated as $R$-module if and only if $J$ contains a Weierstrass polynomial. Indeed, if all elements of $J$ reduce to zero in $\kappa:=k[[X ; \sigma]]$, then $M / \mathfrak{M} M \cong \kappa$ where $\mathfrak{M}:=\operatorname{ker}(: A \rightarrow \kappa)$. But since there is some surjection $R^{n} \rightarrow M$ and $\mathfrak{m} \subseteq \mathfrak{M}$ the module $M / \mathfrak{M} M$ is a finitely generated $k$-module, a contradiction. The other implication is a direct consequence of theorem 3.1.

If, moreover, $J=A f$ is principal, then $A / J$ is a finitely generated $R$-module if and only if $\operatorname{ord}^{\text {red }}(F)$ is finite, i.e. if and only if $A f=A F$ can also be generated by some Weierstrass polynomial $F$.

Of course, there exist right versions for all statements of this section. Indeed, since $\sigma$ is assumed to be an automorphism, one gets a natural ring isomorphism

$$
R\left[[X ; \sigma, \delta] \cong\left[\left[X ; \sigma^{\prime}, \delta^{\prime}\right]\right] R,\right.
$$

where $\sigma^{\prime}=\sigma^{-1}, \delta^{\prime}=-\delta \circ \sigma^{-1}$ and in the latter ring the coefficients are written on the right side of the variable $X$. Moreover, the reduced order is invariant under this isomorphism, i.e. the notion of an distinguished polynomial and its degree is independent of the representation as left or right power series.

\section{Faithful modules}

In this section we calculate the global annihilator ideals of certain modules over the Iwasawa algebra of some $p$-adic Lie group. First we concentrate on the example 2.2, i.e. $A=R[[Y ; \sigma, \delta]]$ with $R=\mathbb{Z}_{p}[[X]]$ or $R=\mathbb{F}_{p}[[X]]$. Recall that in this case $\delta$ is just given as $\sigma$-id. To exclude the case where $G$ respectively $A$ are commutative, we assume that $\epsilon \neq 1$. 
Remark 4.1. (i) There is a canonical isomorphism

$$
R[Y ; \sigma, \delta] \cong R[Z ; \sigma], r \mapsto r, Y \mapsto Z-1
$$

of skew polynomial rings, because

$$
\begin{aligned}
Z r & =(Y+1) r \\
& =Y r+r \\
& =\sigma(r) Y+\delta r+r \\
& =\sigma(r) Y+\sigma(r)-r+r \\
& =\sigma(r)(Y+1)=\sigma(r) Z .
\end{aligned}
$$

(ii) Any ideal $I$ of $A$ which contains a polynomial $0 \neq b=\sum_{i=0}^{s} b_{i} Z^{i}$ also contains a non-zero element $r \in R$. First note that $Z^{i} \gamma=\sigma^{i}(\gamma) Z^{i}$ for $\gamma:=$ $X+1 \epsilon R^{*}$, i.e. $\sigma^{j}(\gamma) Z^{i}-Z^{i} \gamma=\left(\sigma^{j}(\gamma)-\sigma^{i}(\gamma)\right) Z^{i}$ the latter being zero if and only if $i=j$ because $\sigma$ operates without fixpoint on $\Gamma_{1}$. Since $I$ is two-sided it also contains the element

$$
\begin{aligned}
\sigma^{s}(\gamma) b-b \gamma & =\sum_{i=0}^{s} b_{i}\left(\sigma^{s}(\gamma) Z^{i}-Z^{i} \gamma\right) \\
& =\sum_{i=0}^{s-1} b_{i}\left(\sigma^{s}(\gamma)-\sigma^{i}(\gamma)\right) Z^{i}
\end{aligned}
$$

which is nonzero whenever one of the $b_{i}, 0 \leq i \leq s-1$, is nonzero because $R$ is integral. Proceeding recursively one concludes that $I$ contains an element of the form $r Z^{i}$ for some $0 \neq r \in R$ and $i \in \mathbb{N}$. But then it contains also $r$ itself because $Z=Y+1$ is a unit in $A$.

(iii) The same argument proves that the skew Laurent polynomial ring $Q\left[Z, Z^{-1} ; \sigma\right]$ over the maximal ring of quotients $Q$ of $R$ is simple (note that the ring automorphism $\sigma$ extends uniquely to $Q$.)

Proposition 4.2. (i) Let $R=\mathbb{F}_{p}[[X]]$. If $M$ is an A-module which is not torsion as $R$-module, then its annihilator ideal over $A$ vanishes:

$$
\operatorname{Ann}_{A}(M)=0 .
$$

(ii) Now let $R=\mathbb{Z}_{p}[[X]]$. If $M$ is an $A$-module without $p$-torsion and such that the module $M / p$ is not torsion as $R / p=\mathbb{F}_{p}[[X]]$-module (for example if $M$ is a free $R$-module), then $\operatorname{Ann}_{A}(M)=0$.

Recall that an $A$-module $M$ is called faithful if the annihilator ideal $A n n_{A}(M)$ is zero and otherwise $M$ is called bounded. Before proving the proposition we want to draw an immediate conclusion from it in the case $R=\mathbb{Z}_{p}[[X]]$. If $M$ is an $A$-module which is finitely generated as $R$-module and of strictly positive $R$-rank $\operatorname{rk}_{R} M>0$, then the conditions of (ii) in the proposition are satisfied for $N:=M / \operatorname{tor}_{\mathbb{Z}_{p}} M$. Indeed, by [17, cor. 1.11] it holds $\operatorname{rk}_{R / p} N / p N=\operatorname{rk}_{R / p}\left({ }_{p} N\right)+$ $\operatorname{rk}_{R} N=\operatorname{rk}_{R} N \neq 0$ in this case, where ${ }_{p} N$ denotes the kernel of multiplication by p. Thus

Corollary 4.3. Every A-module $M$ which is finitely generated as $R$-module and of strictly positive $R$-rank $\operatorname{rk}_{R} M>0$ is faithful. 
Proof (of prop. 4.9). By the following lemma the second statement is a consequence of the first one, which can be proven as follows. First note that the $R$-torsion submodule $M_{R \text {-tor }}$ of $M$ is an $A$-module, because $X$ is a normal element of $A$ (see below). Thus $M$ has a quotient $N$ without $R$-torsion and since $A n n_{A}(M) \subseteq A n n_{A}(N)$ we may assume without loss of generality that $M$ itself is a torsionfree $R$-module. Now, if $A n n_{A}(M)$ contains a non-trivial element $b \epsilon A$ this can be written as $X^{n} \widetilde{b}$ with $\operatorname{ord}^{r e d}(\widetilde{b})<\infty$ because $R$ is a principal ideal domain all ideals of which are generated by some power of $X$. Due to the Weierstrass preparation theorem $\widetilde{b}$ is a Weierstrass polynomial in the variable $Y$ up to a unit, i.e. we may assume that $b$ is already a Weierstrass polynomial because neither the unit nor $X^{n}$ can annihilate $M$ by assumption. By the above remark then the annihilator ideal contains a nonzero element $r \in R$ which contradicts the assumption that $M$ is a torsionfree $R$-module.

Remark 4.4. By similar arguments one can show that every nonzero two-sided ideal $J$ of $A=\mathbb{F}_{p}[[X, Y ; \sigma, \delta]]$ contains some power of $X$. It follows immediately that for every two-sided ideal $J$ of $A=\mathbb{Z}_{p}[[X, Y ; \sigma, \delta]]$ the cyclic module $A / J$ is finitely generated over $\mathbb{Z}_{p}[[Y]] \cong \Lambda(\Gamma)$ (for any lift $\Gamma \subseteq G$ ). In particular, by an easy induction argument on the number of generators, each finitely generated bounded $A$-module is finitely generated over $\Lambda(\Gamma)$.

Lemma 4.5. Let $\Lambda:=\mathbb{Z}_{p} \llbracket G \rrbracket$ and $\Omega:=\mathbb{F}_{p} \llbracket G \rrbracket \cong \Lambda / p$ the completed group algebras of some pro-finite group $G$ with coefficients in $\mathbb{Z}_{p}$ and $\mathbb{F}_{p}$ respectively and assume that both rings are Noetherian integral domains. If $M$ is an A-module without $p$-torsion then the following holds

$$
\operatorname{Ann}_{\Lambda}(M) \neq 0 \Rightarrow \operatorname{Ann}_{\Omega}(M / p) \neq 0 .
$$

Proof. If $f$ is any nonzero element in $A n n_{\Lambda}(M)$ and $f=p^{n} f^{\prime}$ is the unique factorization such that $f^{\prime}$ is not divisible by $p$ then $f^{\prime}$ is also an element in the annihilator of $M$ because the latter module does not have any $p$-torsion by assumption. Thus the image of $f^{\prime}$ in $\Omega$ is obviously a non-zero element of $A n n_{\Omega}(M / p)$.

In the following we study the behaviour of the annihilator ideal under induction. So let $H$ be a closed subgroup of a $p$-adic Lie group $G$. If $M$ denotes a finitely generated $\Lambda(H)$-module, then we write $\operatorname{Ind}_{G}^{H} M:=\Lambda(G) \otimes_{\Lambda(H)} M$ for the $\Lambda(G)$ module which is induced from $M$.

Let us assume for a moment that $H=\left(h_{1}, \ldots, h_{r}\right)$ is a uniform pro- $p$-group with minimal system of generators $h_{1}, \ldots, h_{r}$. Then, using the isomorphism $\Lambda(H) \cong$ $\prod_{\left(l_{1}, \ldots, l_{r}\right) \in \mathbb{N}^{r}} \mathbb{Z}_{p}\left(h_{1}-1\right)^{l_{1}} \cdots\left(h_{r}-1\right)^{l_{r}}$, it is easily seen that the $\mathbb{Z}_{p}$-linear span $\sum \mathbb{Z}_{p} h_{n}$ is dense in $\Lambda(H)$ where $h_{n}:=h_{1}^{\nu_{1}^{n}} \cdots h_{r}^{\nu_{r}^{n}}$ for some bijection $\mathbb{N} \cong \mathbb{N}^{r}$, $n \rightarrow \nu^{n}=\left(\nu_{1}^{n}, \ldots, \nu_{r}^{n}\right)$. Since every $p$-adic analytic group possesses an uniform subgroup of finite index it follows immediately that there exists (for arbitrary $H$ as above) a countable set $\mathcal{H}=\left\{h_{n} \in H \mid n \in \mathbb{N}\right\}$ such that its $\mathbb{Z}_{p}$-linear span is 
dense in $\Lambda(H)$. For a left ideal $I$ and an element $r$ of a ring $\Lambda$ we will denote by $(I: r):=\{\lambda \epsilon \Lambda \mid \lambda r \in I\}$ the annihilator of $r+I \epsilon \Lambda / I$.

Lemma 4.6. Let $I$ be a left ideal of $\Lambda:=\Lambda(H)$ and set $M:=\Lambda / I$. Then, for any choice of $\mathcal{H}$, the annihilator ideal equals the countable intersection

$$
A n n_{\Lambda}(M)=\bigcap_{n=0}^{\infty} I_{n}
$$

of the left ideals $I_{n}:=I h_{n}^{-1}=\left(I: h_{n}\right)$.

Proof. In general, it holds

$$
\begin{aligned}
\operatorname{Ann}_{\Lambda}(M) & =\bigcap_{m \in M} \operatorname{Ann} n_{\Lambda}(m) \\
& =\bigcap_{r \in \Lambda}(I: r) .
\end{aligned}
$$

Thus $\operatorname{Ann}_{\Lambda}(M)$ is contained in $\bigcap_{n=0}^{\infty} I_{n}$. On the other hand any element $\lambda$ of the latter intersection annihilates $r+I \in M$ for any $r$ in the $\mathbb{Z}_{p}$-linear span of $\mathcal{H}$. Since this span is dense in $\Lambda$ the statement follows by the continuity of the action of $\Lambda$ on $M$.

In order to compare $A n n_{\Lambda(G)}\left(\operatorname{Ind}_{G}^{H} M\right)$ with $\Lambda(G) A n n_{\Lambda(H)}(M)$ the following lemma will be crucial. Note that $\Lambda(G)$ is a flat $\Lambda(H)$-module ([26, proof of lem 5.5]).

Lemma 4.7. Let $I_{i}, i \in \mathbb{N}$, be a set of left ideals in $\Lambda(H)$. Then the following holds:

$$
\Lambda(G) \bigcap_{i} I_{i}=\bigcap_{i} \Lambda(G) I_{i}
$$

A similar statement holds for an arbitrary filtered index set $T$ if the ideals form a descending chain $I_{s} \supseteqq I_{t}$ for $t \geq s$.

Proof. Due to the flatness of $\Lambda(G)$ the functor $\Lambda(G) \otimes_{\Lambda(H)}$ - interchanges with finite intersections. Hence we can replace the ideals $I_{i}$ by the ideals $J_{i}:=\bigcap_{n=0}^{i} I_{n}$ to come into the situation of the second statement, in which the intersection can be expressed as a projective limit: $\bigcap_{T} I_{t}={\underset{\leftarrow}{T}}_{\lim _{t}} I_{t}$. Since inverse limits interchange with the completed tensor product $\Lambda(G) \widehat{\otimes}_{\Lambda(H)}$ - and the latter coincides with the usual tensor product if applied to finitely generated $\Lambda(H)$-modules (see [5]) we obtain 


$$
\begin{aligned}
\Lambda(G) \bigcap_{T} I_{t} & =\Lambda(G) \otimes_{\Lambda(H)} \bigcap_{T} I_{t} \\
& =\Lambda(G) \widehat{\otimes}_{\Lambda(H)} \bigcap_{T} I_{t} \\
& =\Lambda(G) \widehat{\otimes}_{\Lambda(H)}{\stackrel{\lim }{T} I_{t}}_{T} \\
& =\lim _{T} \Lambda(G) \widehat{\otimes}_{\Lambda(H)} I_{t} \\
& =\lim _{T}^{\lim } \Lambda(G) \otimes_{\Lambda(H)} I_{t} \\
& =\lim _{T}^{\lim } \Lambda(G) I_{t} .
\end{aligned}
$$

Proposition 4.8. Let $M$ be a finitely generated $\Lambda(H)$-module. Then the global annihilator ideal of $\operatorname{Ind}_{G}^{H} M$ is contained in ideal generated by the global annihilator of $M$ :

$$
A n n_{\Lambda(G)}\left(\operatorname{Ind}_{G}^{H} M\right) \subseteq \Lambda(G) A n n_{\Lambda(H)}(M) .
$$

In particular, if $M$ is a faithful $\Lambda(H)$-module, then $\operatorname{Ind}_{G}^{H} M$ is a faithful $\Lambda(G)$ module.

Proof. Since a finitely generated $\Lambda(H)$-module $M$ is the sum of finitely many cyclic submodules $M_{i}$ and $A n n_{\Lambda(H)}(M)=\bigcap_{i} A n n_{\Lambda(H)}\left(M_{i}\right)$ this statement is easily reduced to the case of an cyclic module $M=\Lambda(H) / I$. Then $\operatorname{Ind}_{G}^{H} M$ is isomorphic to $\Lambda(G) / \Lambda(G) I$ and it holds

$$
A n n_{\Lambda(G)}(\Lambda(G) / \Lambda(G) I) \subseteq \bigcap_{\mathcal{H}} \Lambda(G) I h^{-1}=\Lambda(G) A n n_{\Lambda(H)}(M)
$$

by the previous lemmata

This result should be compared to a theorem of Harris [16] which tells that the module $\operatorname{Ind}_{G}^{H} \mathbb{Z}_{p}$ is bounded whenever $2 \operatorname{dim} H>\operatorname{dim} G$. Using this proposition we can produce a series of faithful $\Lambda(G)$-modules where $G$ is either an appropriate pro-p-subgroup of $S L_{2}\left(\mathbb{Z}_{p}\right)$ or $G L_{2}\left(\mathbb{Z}_{p}\right)$ (and thus $S L_{n}\left(\mathbb{Z}_{p}\right)$ or $G L_{n}\left(\mathbb{Z}_{p}\right)$ ). Indeed, the closed subgroup $H$ of $G L_{2}\left(\mathbb{Z}_{p}\right)$ generated by the matrices $t=\left(\begin{array}{cc}a & 0 \\ 0 & a^{-1}\end{array}\right)$ and $c=\left(\begin{array}{ll}1 & b \\ 0 & 1\end{array}\right)$, where e.g. $a=1+p$ and $b=1$, i.e. more or less "the" (pro-p-) Borel subgroup of $S L_{2}\left(\mathbb{Z}_{p}\right)$, is isomorphic to the semidirect product $H \cong C \rtimes T$ of $C=c^{\mathbb{Z}_{p}}=\left(\begin{array}{cc}1 & \mathbb{Z}_{p} \\ 0 & 1\end{array}\right)$ and $T=t^{\mathbb{Z}_{p}}$. Hence all the induced 
modules $\operatorname{Ind}_{G}^{H}(\Lambda(H) / \Lambda(H) F)$ where $F$ denotes a distinguished polynomial in $\Lambda(H) \cong \mathbb{Z}_{p}[[X, Y ; \sigma, \delta]]$ are faithful. We should mention that the faithfulness of $\operatorname{Ind}_{G}^{H}(\Lambda(H) / \Lambda(H)(t-1)) \cong \operatorname{Ind}_{G}^{T} \mathbb{Z}_{p}$ was proved by Greenberg (private communication) using "p-adic harmonic analysis."

\section{Modules UP TO PSEUdO-ISOMORPHISM}

In this section let $R$ denote a noetherian local ring with maximal ideal $\mathfrak{m}_{R}$ which is compact with respect to its $\mathfrak{m}_{R}$-adic topology. Furthermore, let $\sigma$ be a ring automorphism and $\delta$ a $\sigma$-derivation as in section 2. We write $A$ for the (compact) ring $R[[Y ; \sigma, \delta]]$ and $B$ for the (non-compact) ring $R[Y ; \sigma, \delta]$. If $\mathfrak{m}_{A}$ denotes the unique maximal ideal of $A$, then $\mathfrak{m}_{B}:=B \cap \mathfrak{m}_{A}$ is a maximal ideal of $B$ and they are the kernels of the canonical $R$-algebra homomorphisms

$$
\begin{aligned}
\mathfrak{m}_{A} & =\operatorname{ker}\left(A=R[[Y ; \sigma, \delta]] \rightarrow R / \mathfrak{m}_{R}\right), \\
\mathfrak{m}_{B} & =\operatorname{ker}\left(B=R[Y ; \sigma, \delta] \rightarrow R / \mathfrak{m}_{R}\right),
\end{aligned}
$$

which are both induced by $Y \mapsto 0$.

Lemma 5.1. With the above notation it holds that

(i) $\mathfrak{m}_{A}=\mathfrak{m}_{R} A+Y A=A \mathfrak{m}_{R}+A Y$ and $\mathfrak{m}_{B}=\mathfrak{m}_{R} B+Y B=B \mathfrak{m}_{R}+B Y$.

(ii) For any $A$-module $M$, the submodules $\mathfrak{m}_{A}^{i} M=\mathfrak{m}_{B}^{i} M$ coincide as $B$-modules. In particular,

$$
\begin{aligned}
& A=\lim _{i} A / \mathfrak{m}_{A}^{i}={\underset{\leftarrow}{\leftarrow}}_{\lim _{i}} B / \mathfrak{m}_{B}^{i} \text { and } \\
& M=\lim _{i} M / \mathfrak{m}_{A}^{i} M={\underset{\iota}{i}}_{\lim _{i}} M / \mathfrak{m}_{B}^{i} M .
\end{aligned}
$$

Proof. Due to compactness and the fact that $\mathfrak{m}_{R}$ is finitely generated over $R$, it is easy to see that $\mathfrak{m}_{R} A=F_{1} A$ and thus $\mathfrak{m}_{R} A+T A=\mathfrak{m}_{R} A+A T=G_{1} A=\mathfrak{m}_{A}$. The proof for $B$ is similar, but easier, while the "left-versions" follow by symmetry. The second item follows immediately from the first one.

By $A$-mod and $B$-mod we denote the category of finitely generated $A$ - and $B$ modules, respectively. For the full subcategories consisting of modules which are even finitely generated over $R$ we write $A$-mod ${ }^{R}$ and $B$-mod ${ }^{R}$, respectively. Similarly, we write mod- $A,{ }^{R} \bmod -A$, etc. for the corresponding categories of right modules.

Lemma 5.2. Let $M$ be in $A$-mod ${ }^{R}$. Then any $B$-submodule $N \subseteq M$ is an $A$ submodule and any $B$-quotient of $M$ is an A-quotient.

Proof. The first statement obviously implies the second one. Since all rings under consideration are noetherian we may assume that $N=B m \subseteq M$ is a cyclic $B$-submodule. Then the $A$-submodule $A m \subseteq M$ is finitely generated over $R$ and thus the left ideal $A n n_{A}(m)$ contains a distinguished polynomial. By the Weierstrass preparation theorem, $A / A n n_{A}(m) \cong B / A n n_{B}(m)$ and the statement for the submodules follows. 
Recall that the grade $j_{A}(M)$ is the minimal integer $i$ such that

$$
\mathrm{E}_{A}^{i} M:=\operatorname{Ext}_{A}^{i}(M, A)
$$

does not vanish.

Lemma 5.3. Let $M$ be in $A-\bmod ^{R}$. Then the following holds:

(i) There is a natural identification of $A$-modules

$$
M=A \otimes_{B} M
$$

where $M$ is considered as $B$-module via restriction. In particular, $A \otimes_{B}-i$ s faithfully flat on the full subcategory of $B-$ mod $^{R}$ whose objects are restrictions of $A-\bmod ^{R}$.

(ii) For all $i$ there is a natural isomorphism of right A-modules

$$
\mathrm{E}_{B}^{i}(M) \otimes_{B} A \cong \mathrm{E}_{A}^{i}(M)
$$

Moreover, $\mathrm{E}_{A}^{i}(M)$ and $\mathrm{E}_{B}^{i}(M)$ are in ${ }^{R}$ mod- $A$ and ${ }^{R}$ mod- $B$, respectively, and $\mathrm{E}_{B}^{i}(M)$ bears a natural right $A$-module structure which extends the right $B$-module structure.

(iii) The grades of $M$ as $A$ - and $B$-module coincide:

$$
j_{A}(M)=j_{B}(M) .
$$

In particular, $M$ is a pseudo-null $A$-module if and only if it is a pseudo-null B-module.

Proof. By [20, Ch. II, prop. 1.8 (3)] there is a natural isomorphism $A \otimes_{B} M \cong$ $\lim _{i} M / \mathfrak{m}_{B}^{i}$. Thus the first statement follows from lemma 5.1. The isomorphism in (ii) is a standard fact of homological algebra using the flatness of $A$ over $B$. If $M$ is finitely generated over $R$ we can find distinguished polynomials $F_{1}, \ldots, F_{n}$ and a surjection

$$
\bigoplus_{i=1}^{r} A / A F_{i} \rightarrow M
$$

Denoting the kernel of it by $N$ the long exact $\mathrm{E}^{\bullet}$-sequence (for both $A$ and $B$ ) gives

$$
0=\mathrm{E}^{0}(N) \rightarrow \mathrm{E}^{1}(M) \rightarrow \bigoplus_{i=1}^{r} \mathrm{E}^{1}\left(A / A F_{i}\right) \rightarrow \mathrm{E}^{1}(N) \rightarrow \mathrm{E}^{2}(M) \rightarrow 0
$$

and $\mathrm{E}^{i}(N) \cong \mathrm{E}^{i+1}(M)$ for all $i \geq 3$. An easy calculation using the "right" version of the Weierstrass preparation theorem shows that

$$
\mathrm{E}_{A}^{1}\left(A / A F_{i}\right) \cong A / F_{i} A \cong B / F_{i} B \cong \mathrm{E}_{B}^{1}\left(B / B F_{i}\right)
$$

as right modules. Thus $E^{1}(M)$ is finitely generated over $R$ and $E_{B}^{1}(M)$ bears a natural right $A$-module structure by lemma 5.2 (indeed its right version). Applying this also to $N$ instead of $M$ we obtain the statement (ii) by induction. Now (iii) follows from (i) and (ii).

Assume now that 
- $R$ is still a compact noetherian local ring with maximal ideal $\mathfrak{m}_{R}$ as before, but now such that

- $g r_{\mathfrak{m}_{R}} R$ is a commutative noetherian regular equidimensional integral domain which is an algebra over some field and

- $\sigma$ induces the identity on the residue class field $k$, i.e. $g r_{\mathfrak{m}_{A}} A=g r_{\mathfrak{m}_{B}} B$ is also commutative.

Then, by 2.10 and its proof, both $A$ and $B$ are Auslander regular and without zero divisor. Thus there is a dimension theory for $A$ - and $B$-modules, for a thorough treatment of which we refer the reader to [10] or [28]. The previous lemma shows that the canonical (co-)dimension filtrations of $M \in A$-mod ${ }^{R}$ considered as $A$ or $B$-module coincide. Recall that $M$ is called pseudo-null if $j_{A}(M) \geq 2$, i.e. if $\mathrm{E}_{A}^{0} M=\mathrm{E}_{A}^{1} M=0$ (similarly for $B$ ). Since $R$ is without zero divisors the set $S:=R \backslash\{0\}$ is an Ore set of $R$ and thus the total ring of quotients $Q:=R_{S}$ exists. By [11, prop. 2.12] $\alpha$ and $\delta$ extend uniquely to $Q$, thus $S$ is also an Ore Set for $B$ and there is a natural isomorphism of rings

$$
C:=B_{S}=Q[X ; \sigma, \delta]
$$

(see below for further explanation in the case where $\delta=\sigma-\mathrm{id}$ ). Then it is well-known that $C$ is a principal ideal domain (division algorithm). Now we are able to prove the following characterization of pseudo-null $A$-modules which are finitely generated over $R$ :

Proposition 5.4. Let $M$ be in $A-\bmod ^{R}$. Then the following holds: $M$ is pseudonull if and only if $\operatorname{rk}_{R} M=0$, i.e. if and only if $M$ is a torsion $R$-module.

Proof. Let $M$ be a pseudo-null $A$-module which is finitely generated as $R$-module. Then

$$
\mathrm{E}_{C}^{i}\left(C \otimes_{B} M\right) \cong \mathrm{E}_{B}^{i}(M) \otimes_{B} C=0
$$

for all $i$ because $C$ has global dimension less or equal to 1 . It follows that $Q \otimes_{R} M \cong$ $C \otimes_{B} M=0$, i.e. $M$ is $R$-torsion. Since $g r_{\mathfrak{m}_{A}} A$ is a commutative noetherian regular equidimensional integral domain which is an algebra over some field, the other direction follows as in [26, lem. 6.4] using Hilbert-Samuel polynomials.

We write $\mathcal{C}$ for the full subcategory of $A$-mod consisting of pseudo-null modules. Since $\mathcal{C}$ is a Serre subcategory the (abelian) quotient category $A$-mod $/ \mathcal{C}$ exists together with a natural functor

$$
q: A-\bmod \rightarrow A-\bmod / \mathcal{C} .
$$

By a sort of homomorphism theorem for abelian categories one may identify the analogous quotient category of $A-\bmod ^{R}$ with a full subcategory of the prior one

$$
A-\bmod ^{R} / \mathcal{C} \cap A-\bmod ^{R} \subseteq A-\bmod / \mathcal{C} .
$$

Henceforth we will denote $\mathcal{C} \cap A-\bmod ^{R}$ just by $\mathcal{C}^{R}$. On the other hand restriction of scalars and tensoring with $C \otimes_{B}-$ gives rise to an exact functor of abelian categories

$$
\Phi: A-\bmod ^{R} \rightarrow B-\bmod ^{R} \rightarrow C-\bmod ,
$$


the kernel of which is just $\mathcal{C}^{R}$ by the above proposition. By the universal property of quotient categories, $A-\bmod ^{R} / \mathcal{C}^{R}$ can be identified with a full subcategory of $C$-mod, which we call by abuse of notation $C$-mod ${ }^{R}$. In terms of left ideals,i.e. of cyclic modules this can be rephrased as follows: Let $\mathcal{R}$ denote the set of non-zero left ideals $I$ such that $M:=A / I$ is in $A-\bmod ^{R}$ (i.e. $I$ contains a distinguished polynomial) and does not contain any non-trivial pseudo-null submodule. By [10, lem. 3.12] $\mathcal{R}$ coincides with the set of all non-zero reflexive left ideals $I$ of $A$ such that $I$ contains a distinguished polynomial. For all $I$ in $\mathcal{R}, I_{B}:=I \cap B$ is a non-zero left ideal of $B$ and its legalization $I_{C}:=\left(I_{B}\right)_{S}=C I_{B}$ is a non-zero left ideal of $C$. Writing $\mathcal{W}$ for the set of all non-zero left ideals of $C$ which contain a distinguished polynomial (with coefficients in $R$ ) we get a map

$$
\begin{aligned}
(-)_{C}: \mathcal{R} & \rightarrow \mathcal{W} \\
I & \mapsto I_{C} .
\end{aligned}
$$

There is also a map in the reverse direction $(-)_{A}: \mathcal{W} \rightarrow \mathcal{R}$, induced by

$$
J \mapsto J_{B}:=J \cap B \mapsto J_{A}:=A J_{B} .
$$

It is easy to see that $\left(J_{A}\right)_{C}=J$ for all $J \in \mathcal{W}$. Indeed, since the left ideals $J_{B} \subseteq J_{A}$ contain a WP the $B$-module $B / J_{B}$ is the restriction of the $A$-module $A / J_{A}$ by lemmas 5.2 and 5.3 (i), i.e. $J_{B}=J_{A} \cap B=\left(J_{A}\right)_{B}$. By [23, prop. 1.15] it follows that $J=C J_{B}=\left(J_{A}\right)_{C}$. On the other hand, it holds $I=\left(I_{C}\right)_{A}$ for any $I$ in $\mathcal{R}$ : using (loc. cit.) again one obtains an inclusion $I_{B} \subseteq C I_{B} \cap B=\left(I_{C}\right)_{B}$ with becomes an equality after scalar extension to $C$. Thus $\left(I_{C}\right)_{A} / I \cong\left(I_{C}\right)_{B} / I_{B}$ is pseudo-null by prop. 5.4 and hence zero by assumption. Thus we have proved the following

Lemma 5.5. $(-)_{C}$ and $(-)_{A}$ are inverse maps, i.e. they are bijections of $\mathcal{R}$ and $\mathcal{W}$.

We shall write $\mathcal{W}_{m}$ and $\mathcal{W}_{d}$ for the subsets of $\mathcal{W}$ consisting of those non-zero left ideals of $C$ which are generated by a monic or distinguished polynomial in $B$, respectively. I am very grateful to R. Sujatha and J. Coates for pointing out to me the following consequence of the above lemma and the resulting question below.

Corollary 5.6. An left ideal $I \triangleleft_{l} A$ is principal if and only if $I_{C}$ belongs to $\mathcal{W}_{m}$. Furthermore, the sets $\mathcal{W}_{m}$ and $\mathcal{W}_{d}$ coincide.

Proof. First we observe that for a monic polynomial $f$ in $B$ the following holds:

$$
C f \cap B=B f .
$$

Indeed, it is easy to see that for monic polynomials there is an Euclidean algorithm both in $B$ and $C$, which implies the claim by the flatness of $Q$ over $R$. Hence, if $J$ is in $\mathcal{W}_{m}$, say $J=C f$, with $f \in B$ monic, we obtain $J_{B}=B f$, and so $J_{A}=A f=A h$, where $h$ is a distinguished polynomial, by the Weierstrass Preparation Theorem. Hence $J=\left(J_{A}\right)_{C}=C h$, i.e. $J$ belongs to $\mathcal{W}_{d}$. If we take $J=I_{C}$, it follows that $I=J_{A}=A h$ is principal. The other implications of the corollary are obvious. 
Hence we are left with the following key question concerning the principality of ideals in $\mathcal{R}$

Question 5.7. Is every non-zero left ideal of $C$ which contains a distinguished polynomial generated by a monic polynomial in $B$ ?

This seems to be a basic question. The answer is yes if and only if every ideal $I$ in $\mathcal{R}$ is principal.

Now, we return to the above quotient category: since simple objects in $A$-mod ${ }^{R} / \mathcal{C}^{R}$ can be represented by some cyclic module $A / I$, one immediately obtains

Theorem 5.8. There exists an equivalence of categories

$$
A-\bmod ^{R} / \mathcal{C}^{R} \cong C-\bmod ^{R},
$$

under which the equivalence classes of simple objects of the quotient category correspond uniquely to equivalence classes of maximal left ideals of $C$ which contain a distinguished polynomial. Furthermore, $C-$ mod $^{R}$ is the smallest abelian subcategory of $C$-mod which is closed under extensions and contains the simple modules corresponding to the latter class of maximal left ideals.

Here the equivalence classes in the quotient category are just the classes of to each other isomorphic objects, while two left ideals $J_{1}, J_{2}$ of $C$ are equivalent if and only if there exists an isomorphism $C / J_{1} \cong C / J_{2}$ of $C$-modules.

We end this section by applying the above result to prime ideals. For simplicity we assume now that $\delta=\sigma-$ id. For any subring $\tilde{B} \subseteq A$ containing the ring $B:=R[Y ; \sigma, \delta] \cong R[Z ; \sigma](Y=Z+1)$ we write $\operatorname{Spec}_{R}(\tilde{B})$ for the set of those prime ideals $\mathfrak{p}$ of $\tilde{B}$ such that $\tilde{B} / \mathfrak{p}$ is a finitely generated $R$-module. Note that the zero ideal is not contained in $\operatorname{Spec}_{R}(\tilde{B})$.

Lemma 5.9. Let $\mathfrak{p}$ be in $\operatorname{Spec}_{R}(A)$. The intersection with $\tilde{B}, \mathfrak{p} \mapsto \mathfrak{p} \cap \tilde{B}$ induces an injective map

$$
\operatorname{Spec}_{R}(A) \rightarrow \operatorname{Spec}_{R}(\tilde{B}) .
$$

Proof. For $\mathfrak{p} \epsilon \operatorname{Spec}_{R}(A), \tilde{B} / \mathfrak{p} \cap \tilde{B}=A / \mathfrak{p}$ by the Weierstrass preparation theorem. Since $\tilde{B}$ is dense in $A$ there is only one $A$-module structure on $\tilde{B} / \mathfrak{p} \cap \tilde{B}$ extending the $B$-module structure which implies injectivity.

Now we specify $\tilde{B}$ to be the skew Laurent polynomial ring

$$
\tilde{B}:=R\left[Z, Z^{-1} ; \sigma\right] \text {. }
$$

Since the ring-automorphism extends uniquely to the skewfield of fractions $Q$ of $R$, the ring $\widetilde{B}$ embeds into

$$
\tilde{C}:=Q\left[Z, Z^{-1} ; \sigma\right]
$$

which is - as legalization of $Q[Z, \sigma]$ - a principal ideal domain. Moreover, $Q\left[Z, Z^{-1} ; \sigma\right]$ is also the quotient $\operatorname{ring} \tilde{B}_{S}$ of $\tilde{B}$ with respect to the set $S=R \backslash\{0\}$ of regular elements of $R$. Indeed, since $\sigma$ is an automorphism, the elements of $R\left[Z, Z^{-1} ; \sigma\right]$ and $Q\left[Z, Z^{-1} ; \sigma\right]$ can be written either with coefficients on the left 
or right site of the powers of $Z$. Let $\mathfrak{p}$ be in $\operatorname{Spec}_{R}(\tilde{B})$ and assume that $\tilde{B} / \mathfrak{p}$ has no $R$-torsion. Then, $\mathfrak{p} \cap R=0$ and thus $\mathfrak{p}_{S} \varsubsetneqq \tilde{B}_{S}=C$ is a proper prime ideal, see [23, prop. 2.1.16]. Denoting by $\operatorname{Spec}_{R, t f}(A)$ the subset of $\operatorname{Spec}_{R}(A)$ consisting of those prime ideals $\mathfrak{p}$ such that $A / \mathfrak{p}$ is without $R$-torsion we obtain

Proposition 5.10. Under the above conditions there is a natural embedding

$$
\operatorname{Spec}_{R, t f}(A) \subseteq \operatorname{Spec}_{R, t f}(\tilde{B}) \subseteq \operatorname{Spec}(\tilde{C}) \backslash\{0\}(\subseteq \operatorname{Spec}(Q[Z ; \sigma]) \backslash\{0\}) .
$$

By $G(A)$ and $G_{R}(A)$ we denote the group of (fractional) $c$-ideals and the subgroup which is generated by $c$-prime ideals in $\operatorname{Spec}_{R, t f}(A)$, respectively. For the definition and a review of its basic properties we refer the reader to [10, §4]. Note that under our current assumptions $A$ is a maximal order (loc. cit. lem. 2.6). Since for any maximal order $\mathcal{O}, G(\mathcal{O})$ is the free abelian group on the set of all prime $c$-ideals, the proposition implies

Corollary 5.11. There is an injective group homomorphism

$$
G_{R}(A) \hookrightarrow G\left(Q\left[Z, Z^{-1} ; \sigma\right]\right)
$$

\section{Completely faithful objects}

In this section we are going to apply the results of the previous ones to certain Iwasawa algebras. In particular we are interested in the existence of bounded and faithful objects in the quotient category.

First we recall some general definitions. For this purpose let $A$ be any maximal order satisfying that every finitely generated torsion $A$-module induces an object of finite length in the quotient category $A-\bmod / \mathcal{C}$. The annihilator ideal of an object $\mathcal{M}$ of $A$-mod $/ \mathcal{C}$ is defined by $\operatorname{Ann}(\mathcal{M}):=\sum_{q(M) \cong \mathcal{M}} A n n_{A}(M)$, where $q$ denotes again the natural functor

$$
q: A-\bmod \rightarrow A-\bmod / \mathcal{C} .
$$

Note that by [27, lem 2.5] in conjunction with [0], cor of thm. 2.5] $\operatorname{Ann}(q(M))=$ $\operatorname{Ann}_{A}\left(M / M_{p s}\right)$ where $M_{p s}$ denotes the maximal pseudo-null submodule of $M$. Recall that the object $\mathcal{M}$ is called completely faithful if $A n n(\mathcal{N})=0$ for any nonzero subquotient $\mathcal{N}$ of $\mathcal{M}$. It is called bounded if $\operatorname{Ann}(\mathcal{N}) \neq 0$ for any subobject $\mathcal{N} \subseteq \mathcal{M}$ (In particular, the image $q(M)$ of any pseudo-null module $M$ is both bounded and completely faithful, by definition).

We should also mention that for a $A$-torsion module $M$, its image $\mathcal{M}:=q(M)$ decomposes uniquely into a direct sum $\mathcal{M} \cong \mathcal{M}_{c f} \oplus \mathcal{M}_{b}$ where $\mathcal{M}_{c f}$ is completely faithful and $\mathcal{M}_{b}$ bounded, cf. [10, prop. 4.1]. Moreover, $\mathcal{M}_{c f}$ or more general all completely faithful objects of finite length $\mathcal{M}$ of $q(A$-mod) are cyclic, i.e. $\mathcal{M} \cong q(\Lambda / L)$ for some non-zero left ideal $L \subseteq \Lambda$ (loc. cit. lemma 2.7 and prop. $4.1)$.

Now let $G=H \rtimes \Gamma$ be an uniform pro- $p$-group as in example 2.3 and set $R:=$ $\Lambda(H)$ as well as

$$
A:=\Lambda(G) \cong \Lambda(H)[[Y ; \sigma, \delta]]=R[[Y ; \sigma, \delta]]
$$


For some left ideal $I$ of $A$, consider the cyclic $A$-module $M=A / I$ and assume that it is finitely generated as $R$-module and has no non-zero pseudo-null submodule. Then, by proposition 5.4, $M$ is torsionfree as $R$-module. We shall denote the annihilator ideal of $M$ by $J=A n n_{A}(M)$.

Lemma 6.1. In the above situation, assume that $M$ is bounded, i.e. $J \neq 0$. Then the module $\Lambda / J$ is finitely generated over $R$, too, and has no non-zero pseudo-null submodule, either.

Proof. We use the same notations as in lemma 4.6 and 4.7 ( $\Lambda$ being replaced by $A$ ). Since $I_{n}=I h_{n}^{-1}$ and $h_{n}$ is a unit in $A$, obviously $A / I_{n} \cong A / I$ and we obtain an injection

$$
A / J_{i} \subseteq \prod_{n=0}^{i} A / I_{n} \cong \prod M .
$$

Thus, for all $i \geq 0$, the module $A / J_{i}$ has no non-zero pseudo-null submodule. Now, assume that infinitely many subquotients $J_{i} / J_{i+1}$ of the chain of left ideals

$$
\cdots \subseteq J_{i+1} \subseteq J_{i} \subseteq \cdots \subseteq J_{1} \subseteq J_{0}=I
$$

are non trivial (here we assume without loss of generality that $h_{0}=1$, i.e. $J_{0}=$ $\left.I_{0}=I\right)$. Then, since $0 \neq J_{i} / J_{i+1} \subseteq A / J_{i+1}$ cannot be pseudo-null, the image $q\left(J_{i} / J_{i+1}\right)$ would be non-zero for infinitely many $i$ contradicting the fact that all torsion $A$-modules have finite length in the quotient category. Thus the above chain becomes stationary, say $J=J_{k}$, and hence

$$
A / J=A / J_{k} \subseteq \prod_{n=0}^{k} M
$$

is finitely generated over $R$ and without non-zero pseudo-null submodule.

This lemma tells us that the identification

$$
A-\bmod ^{R} / \mathcal{C}^{R} \cong C-\bmod ^{R}
$$

in theorem 5.8 respects "boundedness", i.e. an object $\mathcal{M}=q(M)$ is bounded if and only if $C \otimes_{B} M$ is a bounded $C$-module. In particular, $q(A / I)$ is bounded if and only if $I_{C}$ is a bounded ideal of $C$, i.e. contains a nonzero ideal. Also, using [10, prop. 4.4], it implies that $G_{R}(A) \cong G\left(\left(A-\bmod ^{R} / \mathcal{C}^{R}\right)^{b}\right)$ where the latter denotes the Grothendieck group of the full subcategory of bounded objects $\left(A-\bmod ^{R} / \mathcal{C}^{R}\right)^{b}$ of $A-\bmod ^{R} / \mathcal{C}^{\mathcal{R}}$. Hence, by the results of the previous section we obtain the following

Proposition 6.2. There is an injective group homomorphism

$$
G\left(\left(A-\bmod ^{R} / \mathcal{C}^{R}\right)^{b}\right) \hookrightarrow G\left(Q\left[Z, Z^{-1} ; \sigma\right]\right) .
$$


There are two special cases of particular interest. First, if $G$ is the semidirect product of two copies of $\mathbb{Z}_{p}$, we have seen that $Q\left[Z, Z^{-1} ; \sigma\right]$ is simple (cf. remark 4.1), thus $G\left(Q\left[Z, Z^{-1} ; \sigma\right]\right)=0$ and we obtain the following

Theorem 6.3. Let $G$ be a non-abelian group isomorphic to $\mathbb{Z}_{p} \rtimes \mathbb{Z}_{p}$. Then the image $q(M)$ of any $M \in A-$ mod $^{R}$, is completely faithful, i.e. the subcategory $q\left(A-\bmod ^{R}\right)$ of $A-\bmod / \mathcal{C}$ consists only of completely faithful objects. In particular, all objects $\mathcal{M}$ of $q\left(A-\bmod ^{R}\right)$ are cyclic, i.e. $\mathcal{M} \cong q(\Lambda / L)$ for some non-zero left ideal $L \subseteq \Lambda$.

Of course, the theorem is also an immediate consequence of corollary 4.3 and proposition 6.5.

Second, if $G$ is an open subgroup of $G L_{2}\left(\mathbb{Z}_{p}\right)$ such that $G=H \times \Gamma$ where $H=G \cap S L_{2}\left(\mathbb{Z}_{p}\right)$ and $\Gamma=\operatorname{center}(G)$, then $\sigma$ is the identity, i.e. the variable $Z$ or $Y$ commute with $Q$. It is well known and easy to check that then every twosided ideal of $Q[Y]$ can be generated by an element in $Z(Q)[Y] \subseteq Q[Y]$ where $Z(Q)$ denotes the center of the skewfield $Q$. In $[18$ S. Howson has proved that $Z(R)=\mathbb{Z}_{p}$ in this case. In order to determine the bounded $A$-modules which are finitely generated over $R$ and not pseudo-null it might be crucial to answer the following

Question 6.4. Let $H$ be any open pro-p-subgroup of $S L_{2}\left(\mathbb{Z}_{p}\right)$. Is the center $Z(Q)$ of the skewfield of fractions $Q$ of $\Lambda(H)$ (i) equal to $\mathbb{Q}_{p}$, (ii) algebraic over $\mathbb{Q}_{p}$ or (iii) has it transcendent elements over $\mathbb{Q}_{p}$ ? Are the elements of $Q \backslash Z(Q)$ algebraic or transcendent over $Z(Q)$ ?

In any case, we know that the simple objects which can be represented by $A$ modules which are finitely generated over $R$ correspond to those maximal left ideals of $Q[Y]$ which contain a distinguished polynomial. If item (i) of the question holds it would probably tell us that the only bounded objects are those which are induced from the center of $G$.

Since $\Lambda(H) \subseteq \Lambda(G)$ for the canonical normal subgroup $H$ of $G$, we may consider any $\Lambda(G)$-module $M$ also as $\Lambda(H)$-module. If such $M$ is even finitely generated over $\Lambda(H)$, then it is known that $\operatorname{dim}_{\Lambda(H)} M \geq \operatorname{dim}_{\Lambda(G)}$ ([26, lem. 6.4]). We now will prove that in the case where $G \cong \mathbb{Z}_{p} \rtimes \mathbb{Z}_{p}$. equality holds.

Proposition 6.5. Let $G \cong \mathbb{Z}_{p} \rtimes \mathbb{Z}_{p}$. With the above notations it holds that $\operatorname{dim}_{\Lambda(H)} M=\operatorname{dim}_{\Lambda(G)}$. In particular, $M$ is a pseudo-null $\Lambda(G)$-module if and only if it is a torsion $\Lambda(H)$-module.

Thus a pseudo-null $\Lambda(G)$-module which is finitely generated over $\Lambda(H)$ is - as $\Lambda(H)$-module up to finite modules - the direct sum of a finitely generated $\mathbb{Z}_{p}$ -module and summands of the form $\mathbb{Z} / p^{n}[[H]]$.

Proof. Since $M$ is zero-dimensional (as $\Lambda(\mathrm{G})$ - or $\Lambda(\mathrm{H})$-module) if and only if it is finite, we only have to prove

Claim: If $M$ is torsion-free as $\Lambda(\mathrm{H})$-module than it is not a pseudo-null module. 
This is just the statement of proposition 5.4, but we want to give a different argument which might be interesting on its own: Since $X$ is a normal element of $\Lambda(\mathrm{G}), M$ has a descending chain of submodules $\left\{X^{n} M\right\}_{n \in \mathbb{N}}$. If $M$ is torsionfree as $\Lambda(H) \cong \mathbb{Z}_{p}[[X]]$-module, then it es easy to see that the subquotients $X^{n+1} M / X^{n} M$ are isomorphic to $M / X M$ as $\mathbb{Z}_{p}$-module. Since the latter module cannot be finite by the strong Nakayama lemma, it follows that these subquotients have dimension 1 both over $\Lambda(\mathrm{G})$ and $\Lambda(\mathrm{H})$ (see 28, prop. 3.5 (ii), cor. 4.8]). Choosing a good filtration on $M$ (and taking the induced filtrations on $\left.X^{n} M\right)$ we get an infinite descending chain of associated graded modules

$$
\operatorname{gr} M \supsetneqq g r(X M) \supsetneqq \cdots \supsetneqq g r\left(X^{n} M\right) \supsetneqq \cdots
$$

with subquotients $\operatorname{gr}\left(X^{n} M\right) / g r\left(X^{n+1} M\right) \cong \operatorname{gr}\left(X^{n} M / X^{n+1} M\right)$ of Krull dimension 1 over $\operatorname{gr} \Lambda(G) \cong \mathbb{F}_{p}\left[X_{0}, X_{1}, X_{2}\right]$ by [28, thm. 3.22]. This implies that $\operatorname{gr} M$ has Krull dimension 2, i.e. $\operatorname{dim}_{\Lambda(G)} M=2$, by the next lemma.

Lemma 6.6. Let $R$ be a commutative Noetherian ring with finite Krull dimension, $M$ a finitely generated $R$-module which has an infinite chain of submodules

$$
M=M_{0} \supsetneqq M_{1} \supsetneqq \cdots \supsetneqq M_{i} \supsetneqq \cdots
$$

with non-zero subquotients $M_{i} / M_{i+1}$ of Krull-dimension $\alpha$. Then, the Krull dimension of $M$ is (strictly) greater than $\alpha$.

Proof. Assume the contrary. Then $\operatorname{deg}(M):=\sum_{\operatorname{dim} R / \mathfrak{p}=\alpha}$ length $_{A_{\mathfrak{p}}} M_{\mathfrak{p}}$ is finite (compare $₫$, VIII $\S 1.5]$ ). Since the function deg is additive (loc.cit.), all subquotients contribute a nonzero positive number to $\operatorname{deg}(M)$ by assumption, a contradiction.

Remark 6.7. The lemma corresponds to the more or less well known fact that for a commutative Noetherian ring the classical Krull-dimension coincides with the Gabriel-dimension or, depending on the definition, with the Gabriel-dimension minus one, see the notes "Krull dimension" in [14, p. 239].

Question 6.8. For which p-adic Lie groups without p-torsion, does it hold that the Gabriel-dimension of every finitely generated $\Lambda(G)$-module coincides with its Bjoerk-dimension, respectively the Krull-dimension of the associated graded module?

Of course the question is answered positively for all abelian p-adic Lie groups without $p$-torsion.

\section{Unique Factorisation Rings}

Chatters and Jordan studied in [9] a class of Noetherian (= left and right Noetherian) rings which in the commutative case consists of the Noetherian unique factorisation domains: 
A prime Noetherian ring $A$ is called unique factorisation ring (UFR) if every non-zero prime ideal of A contains a non-zero principal prime ideal.

If $A$ satisfies the descending chain condition on prime ideals (e.g. if the classical Krull dimension $\operatorname{dim}(\operatorname{Spec}(A))$ of $A$ is finite, where $\operatorname{Spec}(A)$ is the poset of prime ideals, see [14, Appendix 3.]), then the above condition is equivalent to

\section{Every height-1 prime ideal of $A$ is principal.}

The meaning of principal ideal $I$ is that $I=a A=A a$ for some element $a \epsilon A$. Such elements are called normal and if $I$ is a prime ideal in addition than $a$ is called prime element. The class of UFRs contains the following much more restricted one (Remark (5) in [9]):

A Noetherian integral domain A which contains at least one height-1 prime ideal is called unique factorisation domain (UFD) if every height-1 prime $P$ of $A$ is principal and $A / P$ is an integral domain.

We also should mention that any UFR $A$ is a unique factorisation ring in the sense of [ [0], i.e. $A$ is a maximal order and any prime $c$-ideal - a prime ideal which is (left or right) reflexive - is principal (cf. the remark after prop. 1.5 in [1]).

Now we come back to our example 2.2, where $A=\mathbb{F}_{p} \llbracket G \rrbracket$ is the completed group algebra of the semidirect product $G=\mathbb{Z}_{p} \rtimes \mathbb{Z}_{p}$. It seems that $A$ is the first example of a complete respectively power series ring which admits unique factorisation.

Theorem 7.1. The ring $A=\mathbb{F}_{p} \llbracket G \rrbracket=\mathbb{F}_{p}[[X, Y ; \sigma, \delta]]$ is a UFD. Furthermore, $(X):=A X A$ is the only prime ideal of height one in $A$ and $X$ is - up to multiplication by units - the only prime element of $A$.

Proof. Since $(X)$ is the kernel of

$$
{ }^{-}: \mathbb{F}_{p}[[X, Y ; \sigma, \delta]] \rightarrow \mathbb{F}_{p}[[Y]]
$$

it is a prime ideal which coincides with $X A$ and by symmetry also with $A X$ (if the coefficients are written on the right of the powers of $Y$ ). By the principal ideal theorem [23, thm. 4.1.11] $(X)$ has height 1 . Now assume that there is some height-1 prime ideal $P$ of $A$ different from $(X)$. Then $P$ contains an element $p$ with $\bar{p} \neq 0$ in $\mathbb{F}_{p}[[Y]]$. By the Weierstrass preparation theorem $p$ is in $R[Y ; \sigma, \delta]$ up to a unit where $R=\mathbb{F}_{p}[[X]]$. The remark 4.1 (ii) implies that $P \cap R \neq 0$, i.e. $X^{n} \subseteq P \cap R \subseteq P$ for some $n$ because all ideals of $R$ are of the form $\left(X^{n}\right)$. Since $P$ is prime it follows that $(X) \subseteq P$, a contradiction. Thus the unique height-1 prime $(X)$ is principal with $A /(X)$ being integral, i.e. $A$ is a UFD.

Similarly, it is seen that $X^{n}, n \in \mathbb{N}$, are - up to multiplication by units - the only normal elements of $A$.

Let $C(X)$ be the set of elements of $A$ which are regular modulo $(X)$ and $S=$ $\left\{X^{n} \mid n \in \mathbb{N}\right\}$. Then it is known (and in this example easy to verify) that the classical (left and right) localisations $A_{(X)}:=A_{C(X)}$ and $A_{S}$ of $A$ with respect to $C(X)$ and $S$ exist. They have the following properties

(i) $A_{(X)}$ is a bounded local principal ideal domain with a single prime ideal $X A_{(X)}$ and whose only (left or right or two-sided) ideals are of the form 
$X^{n} A_{(X)}$ (see 8$]$ prop. 2.8 and its proof in conjunction with th. 2.7, compare also with [6, prop. 2.5]).

(ii) $A_{S}$ is simple ([9, lem. 2.1]).

(iii) $A=A_{(X)} \cap A_{S}([9$, thm. 2.3]). This should be compared also with Chamarie's result 3.3-3.5 in [7]: While $A_{S}$ is "responsible" for the faithful modules the bounded ring $A_{(X)}$ covers the bounded modules (in the quotient category of $A$-mod modulo the Serre subcategory of pseudo-null $A$-modules, see [10] or [28]).

Remark 7.2. Let $A=\mathbb{F}_{p} \llbracket G \rrbracket$ and $J$ be a left ideal of $A$ such that $M:=A / J$ does not contain any pseudo-null submodule. Then $M$ is faithful in the quotient $A$-mod $/ \mathcal{C}$ if and only if no power $X^{n}, n \in \mathbb{N}$, is contained in $J$. Indeed, under the assumptions the annihilator of the image of $M$ in the quotient category coincides with the usual annihilator ideal $A n n_{A}(M)$. If $M$ is bounded, the latter ideal is a $c$-ideal in $A$, i.e. a power of the unique prime ideal $(X)$ ([10, lem 4.3]). In particular, $X^{n}$ is contained in $J$ for some $n$. On the other hand, assume that $X^{n} \in J$. Then $J$ contains obviously the two-sided ideal $A X^{n}$.

We should also mention that the fact that $\mathbb{F}_{p} \llbracket G \rrbracket$ is a UFR in the sense of $\llbracket$ 1 can be derived more easily from the following general theorem:

Theorem 7.3. Let $A$ be a local noetherian integral ring which is a maximal order (in its ring of quotients). If the global homological dimension of $A$ is less or equal to 2 then $A$ is a UFR in the sense of [1].

Proof. Conferring prop. 1.8. (2) in [1] it is sufficient to prove that every reflexive ideal $I$ is principal: Denote by $I^{+}:=\operatorname{Hom}_{A}(I, A)$ the dual of $I$ and choose a projective resolution of $\mathrm{I}^{+}$

$$
0 \rightarrow P_{1} \rightarrow P_{0} \rightarrow I^{+} \rightarrow 0 .
$$

Applying the functor $-^{+}$we obtain an exact sequence

$$
0 \rightarrow I \rightarrow P_{0}^{+} \rightarrow P_{1}^{+} \rightarrow \operatorname{Ext}_{A}^{1}\left(I^{+}, A\right) \rightarrow 0
$$

because $I \cong I^{++}$is reflexive. Now it follows by homological algebra that $I$ has projective dimension equal to zero (since the projective dimension of $\operatorname{Ext}_{A}^{1}\left(I^{+}, A\right.$ ) is less or equal to 2). But as $A$ is local it follows that $I$ is a free left $A$-module. By symmetry it is also a right principal ideal and the theorem follows.

Recall that a principal ideal domain $(P I D)$ is an integral ring all left and right ideals of which are principal.

Corollary 7.4. Let $R$ be a local PID and $\pi$ a generator of the unique maximal ideal $\mathfrak{m}=(\pi)$ of $R$ and assume that $R$ is complete in its $\pi$-adic topology. If $\sigma$ is a ring endomorphism of $R$ which induces an automorphism $\bar{\sigma}$ of $\kappa:=R /(\pi)$, then, for any $\sigma$-derivation $\delta$ as in section $\mathbb{Q}$, the skew power series ring $R[[Y ; \sigma, \delta]]$ is an UFD in the sense of [1]. 
Proof. Since $\kappa$ is a skew field its global dimension is zero. Hence, by corollary 2.10 and [23, thm. 7.5.3 (iii)], $\mathrm{gl} A \leq \mathrm{gl} g r_{\pi} R+1=\mathrm{gl} \kappa[x]+1=2$ and the result follows from proposition 2.11, corollary 2.10 (iii) and the theorem.

In general, there is a canonical homomorphism

$$
G(R[Y ; \sigma, \delta]) \rightarrow G(R[[Y ; \sigma, \delta]])
$$

induced by $I \rightarrow \widehat{I}:=A \otimes_{B} I \cong I \otimes_{B} A$. In those cases where this map is surjective (e.g. for $\mathbb{F}_{p} \llbracket G \rrbracket$ as above) the UFR property for $B$ in the sense of [1] implies the UFR property for $A$ (supposed the latter ring is a maximal order). Anyway, one obtains the following implication

Proposition 7.5. Let $R$ be a compact noetherian local ring with maximal ideal $\mathfrak{m}_{R}$. If $R[Y ; \sigma, \delta]$ is a UFR in the sense of [1], then all ideals in $G_{R}(A)$ are principal.

We should mention that theorem 2.4 and proposition 1.8 (2) of [1] imply the following: If a noetherian ring $R$ is a UFR in their sense, then so is $R[Z, \sigma]$. E.g., if $\Lambda(H)$ is a UFR, then all ideals in $G_{\Lambda(H)}(\Lambda(G))$ are principal, where $H \subseteq G$ are the groups of example 2.3 or in particular of example 2.4 where $H \subseteq G \subseteq G L_{2}\left(\mathbb{Z}_{p}\right)$.

Proof. Let $I$ be a prime $c$-ideal of $A$ such that $A / I$ is finitely generated over $R$, set $I_{B}:=B \cap I$ as before and consider the following exact and commutative diagram

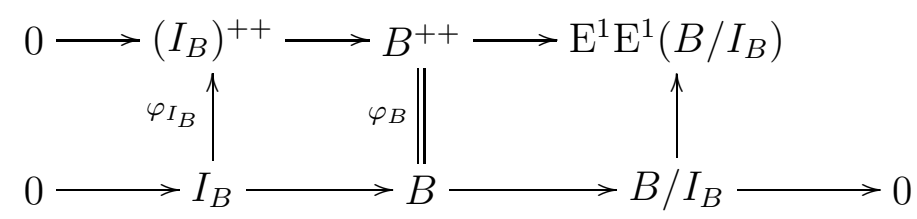

Here, the first line is easily obtained by forming twice the long exact $\mathrm{E}^{\bullet}$-sequence of the bottom line and the vertical homomorphisms are (induced by) the canonical maps from a module to its bi-dual. By the snake lemma, lemma 5.2 and 4.3 (i), the functor $A \otimes_{B}-$ is faithfully flat on the cokernel $W$ of $\varphi_{I_{B}}$. But $A \otimes_{B} W \cong$ $\operatorname{cokern}\left(\varphi_{I}\right)$ (as $A$-module) is zero by assumption. Thus, by left-right symmetry, $I_{B}$ is also a reflexive ideal and maps onto $I$, i.e. the following restriction of the above homomorphism is surjective

$$
G_{R}(B) \rightarrow G_{R}(A) .
$$

The result follows.

In section 4 we have seen that $\Lambda(G)$ possesses an abundance of faithful modules. Now we will describe an infinite series of bounded modules which induces an infinite series of pairwise non-isomorphic simple objects in the quotient category by pseudo-null modules. Indeed, by [10, prop. 4.4] the prime $c$-ideals, i.e. those prime ideals which are reflexive (both as left and right $\Lambda(G-)$ module), correspond to the annihilator ideals of bounded simple objects. Thus it is sufficient to find an infinite series of prime ideals which are principal and thus reflexive. 
To this end we set $\omega_{-1}:=1, \omega_{n}:=(X+1)^{p^{n}}-1, n \geq 0$, and denote the cyclotomic polynomial by

$$
\xi_{n}:=\frac{\omega_{n}}{\omega_{n-1}}, \quad n \geq 0
$$

Then, $\xi_{0}=\omega_{0}=X$, while

$$
\xi_{n}=\sum_{i=0}^{p-1}(1+X)^{i p^{k-1}}, \quad k \geq 1 .
$$

Proposition 7.6. The polynomials $\xi_{n}$ are prime elements of $\Lambda=\Lambda(G)$, i.e. $\left(\xi_{n}\right):=\Lambda \xi_{n}=\xi_{n} \Lambda$ is a prime ideal of $\Lambda$ for every $n \geq 0$. Furthermore, the quotient $\Lambda /\left(\xi_{n}\right)$ is isomorphic to the integral ring $\mathbb{Z}_{p}\left[\zeta_{p^{n}}\right][[Y ; \bar{\sigma}, \bar{\delta}]]$, where $\zeta_{p^{n}}$ denotes a primitive $p^{n}$-root of unit and $\bar{\sigma}$ and $\bar{\delta}$ are induced by $\sigma$ and $\delta$, respectively.

In fact, using the integrality of $\mathbb{Z}_{p}\left[\zeta_{p^{n}}\right][[Y ; \bar{\sigma}, \bar{\delta}]]$ and the change of rings spectral sequence for Ext-groups, one can prove that the $\Lambda$-modules

$$
\Lambda /\left(\xi_{n}\right) \cong \mathbb{Z}_{p}\left[\zeta_{p^{n}}\right][[Y ; \bar{\sigma}, \bar{\delta}]]
$$

themselves induce simple objects $q\left(\Lambda /\left(\xi_{n}\right)\right)$ in the quotient category. Note that, in general, a prime $c$-ideal $\mathfrak{p}$ is only the largest two-sided ideal in some reflexive left ideal $I$ such that $q(\Lambda / I)$ is simple (with annihilator ideal $\mathfrak{p}$ ).

Proof. First, we show that $\omega_{m}$ and $\xi_{n}$ are normal elements in $\Lambda$ : Since $\omega_{n} \Lambda=$ $\prod \mathbb{Z}_{p}[[X]] \omega_{n} Y^{i}$ is the kernel of the canonical map

$$
\mathbb{Z}_{p}[[X, Y ; \sigma, \delta]] \cong \Lambda(G) \rightarrow \Lambda\left(\left(H / H_{n}\right) \rtimes \Gamma\right) \cong\left(\mathbb{Z}_{p}[[X]] / \mathbb{Z}_{p}[[X]] \omega_{n}\right)[[Y ; \bar{\sigma}, \bar{\delta}]],
$$

this is a two-sided ideal, which by symmetry coincides with $\Lambda \omega_{n}$, thus $\omega_{n}$ is normal. Hence, we obtain

$$
\omega_{n-1} \xi_{n} \Lambda=\omega_{n} \Lambda=\Lambda \omega_{n}=\Lambda \omega_{n-1} \xi_{n}=\omega_{n-1} \Lambda \xi_{n}
$$

i.e. $\xi_{\Lambda}=\Lambda \xi_{n}$ because $\Lambda$ does not have zero divisors.

Since $\left(\mathbb{Z}_{p}[[X]] / \mathbb{Z}_{p}[[X]] \xi_{n}\right)[[Y ; \sigma, \delta]] \cong \mathbb{Z}_{p}\left[\zeta_{p^{n}}\right][[Y ; \sigma, \delta]]$ is integral by cor. 2.10 (note that $g r \mathbb{Z}_{p}\left[\zeta_{p^{n}}\right] \cong \mathbb{F}_{p}[\pi]$, where $\pi$ is the image of $X$ respectively $\left.\zeta_{p^{n}}-1\right)$.

\section{The "False Tate Curve"}

In this section we will study a Galois extension of a number field $k$ which arises from a $p$-adic Galois representation which is analogous to the local presentation induced by a Tate elliptic curve. Thus, following J. Coates, we call it "false Tate curve."

So let $\alpha \epsilon k^{\times}$be a unit of $k$ which is not a root of unity. Consider the discrete $G_{k^{-}}$ module $A:=\bar{k}^{\times} / \Delta_{\alpha}$, where $\bar{k}$ denotes a fixed algebraic closure of $k, G_{k}=G(\bar{k} / k)$ the absolute Galois group of $k$ and $\Delta_{\alpha}=\alpha^{\mathbb{Z}}$ the subgroup of $\bar{k}^{\times}$which is generated by $\alpha$. Then the $p^{n}$-division points $A_{p^{n}}$ of $A$ contain the $p^{n}$-roots of unity $\mu_{p^{n}} \subseteq \bar{k}^{\times}$ and we have natural exact sequences of $G_{k}$-modules for every $n$

$$
0 \rightarrow \mu_{p^{n}} \rightarrow A_{p^{n}} \rightarrow \mathbb{Z} / p^{n} \mathbb{Z} \rightarrow 0
$$


where the latter module has trivial $G_{k}$ action and where the surjection $A_{p^{n}} \rightarrow$ $\mathbb{Z} / p^{n} \mathbb{Z}$ is given as follows: If $\beta \epsilon \bar{k}^{\times}$represents an element of $A_{p^{n}}$, i.e. $\beta^{p^{n}}=\alpha^{c}$ for some $c \in \mathbb{Z}$, then its class $\beta \Delta_{\alpha}$ is send to $c \bmod p^{n}$.

Writing $T_{p}(B):=\underbrace{\lim }_{n} B_{p^{n}}$ the Tate-module and $V_{p}(B):=T_{p}(B) \otimes_{\mathbb{Z}_{p}} \mathbb{Q}_{p}$ for the $p$ adic representation associated with an arbitrary discrete $G_{k}$ module $B$, we obtain the exact sequences

$$
0 \rightarrow \mathbb{Z}_{p}(1) \rightarrow T_{p}(A) \rightarrow \mathbb{Z}_{p} \rightarrow 0
$$

where $\mathbb{Z}_{p}(1)=T_{p}(\mu)$ is the first Tate-twist of $\mathbb{Z}_{p}$, and

$$
0 \rightarrow \mathbb{Q}_{p}(1) \rightarrow V_{p}(A) \rightarrow \mathbb{Q}_{p} \rightarrow 0
$$

If $G$ denotes the kernel of the action $\rho: G_{k} \rightarrow G L(V)$ of $G_{k}$ on $V=V_{p}(A)$ :

$$
G:=\operatorname{ker}\left(\rho: G_{k} \rightarrow G L(V)\right) ;
$$

then the field $k_{\infty}:=\bar{k}^{G}$ which is determined by $G$ via Galois theory is of course $k\left(A_{p^{\infty}}\right)=k\left(\mu_{p^{\infty}}, \alpha^{p^{-\infty}}\right)$, the field, which arises by adjoining the $p$-power roots of unity as well as $p$-power roots of $\alpha$ to $k$.

For the rest of the paper, we assume that $k$ contains the $p$-th roots of unity $\mu_{p} \subseteq k$. By $k_{c y c l}$ we denote the cyclotomic $\mathbb{Z}_{p}$-extension $k_{c y c l}=k\left(\mu_{p}\right)$ of $k$, by $H:=G\left(k_{\infty} / k_{c y c l}\right)$ and $\Gamma:=G\left(k_{c y c l} / k\right)$ the Galois groups of the extensions $k_{\infty} / k_{\text {cycl }}$ and $k_{\text {cycl }} / k$ respectively. Then Kummer theory tells us that

$$
\operatorname{Hom}\left(H, \mu_{p^{\infty}}\right) \cong \operatorname{im}\left(\Delta_{\alpha} \otimes_{\mathbb{Z}} \mathbb{Q}_{p} / \mathbb{Z}_{p} \rightarrow k_{c y c l}^{\times} \otimes_{\mathbb{Z}} \mathbb{Q}_{p} / \mathbb{Z}_{p}\right) \cong \mathbb{Q}_{p} / \mathbb{Z}_{p}
$$

as $G_{k}$-modules. In particular, $H \cong \mathbb{Z}_{p}(1)$ as $\Gamma$-module, i.e. $G$ is isomorphic to the (non-abelian) semi-direct product

$$
G \cong H \rtimes \Gamma=\mathbb{Z}_{p}(1) \rtimes \mathbb{Z}_{p}
$$

Of course, the arithmetic in $k_{\infty} / k$ depends decisively on the choice of $\alpha$. If we take $\alpha=p$ for example we have the following properties.

Lemma 8.1. Let $k=\mathbb{Q}\left(\mu_{p}\right)$ and $k_{\infty}=k\left(\mu_{p^{\infty}}, p^{p^{-\infty}}\right)$. Then the extension $k_{\infty} / k$ is

(i) totally ramified at the unique place over $p$, in particular there is just one prime of $k_{\infty}$ above $p$.

(ii) unramified outside $p$.

Proof. (The proof arose from a discussion with Y. Hachimori) It suffices to prove these statements for the finite extensions $k\left(\mu_{p^{n}}, p^{p^{-n}}\right.$.) Then item (ii) follows from [2, Lemma 5].

Now consider the local extensions $K=\mathbb{Q}_{p}\left(\mu_{p^{n}}\right)$ and $L=K\left(p^{p^{-n}}\right)$ of $\mathbb{Q}_{p}$. Since the extension $\mathbb{Q}_{p}\left(p^{p^{-1}}\right) / \mathbb{Q}_{p}$ is not Galois, no $p$ th root of $p$ can be contained in the cyclic extension $K / \mathbb{Q}_{p}$. Hence, it follows from Kummer theory that the degree of $L$ over $K$ is $[L: K]=p^{n}$, i.e. $\left[L: \mathbb{Q}_{p}\right]=\left[\mathbb{Q}\left(\mu_{p^{n}}, p^{p^{-n}}\right): \mathbb{Q}\right]\left(=(p-1) p^{2 n-1}\right)$ and 
in particular $p$ does not split in $k\left(\mu_{p^{n}}, p^{p^{-n}}\right)$. Since the maximal abelian quotient $G^{a b}$ of $G=G\left(L / \mathbb{Q}_{p}\right) \cong G(L / K) \rtimes G\left(K / \mathbb{Q}_{p}\right)$ is isomorphic to

$$
G^{a b} \cong G(L / K)_{G\left(K / \mathbb{Q}_{p}\right)} \oplus G\left(K / \mathbb{Q}_{p}\right)=G\left(K / \mathbb{Q}_{p}\right)
$$

(note that $G(L / K) \cong \mathbb{Z} / p^{n}(1)$ has no non-zero $G\left(K / \mathbb{Q}_{p}\right)$-invariant quotient because $G\left(K / \mathbb{Q}_{p}\right)$ acts via the cyclotomic character on $\left.G(L / K)\right)$, the only cyclic extensions of $\mathbb{Q}_{p}$ in $L$ are contained in $K$ and cannot be unramified. Hence $p$ is totally ramified in $k\left(\mu_{p^{n}}, p^{p^{-n}}\right)$ for all $n$.

Let $L$ be the maximal unramified abelian $p$-extension of $k_{\infty}$. Then its Galois group $X_{n r}=G\left(L / k_{\infty}\right)$ is a compact $\Lambda(G)$-module where $\Lambda(G)$ denotes the Iwasawa algebra

$$
\Lambda(G)=\mathbb{Z}_{p} \llbracket G \rrbracket \cong \mathbb{Z}_{p}[[X, Y ; \sigma, \delta]] .
$$

If we define $H_{n}$ and $\Gamma_{n}$ to be the unique subgroup of $H$ and $\Gamma$ of index $p^{n}$, respectively, then $G_{n}:=H_{n} \rtimes \Gamma_{n}$ is an open normal subgroup of $G$ of index $p^{2 n}$. Let us write $k_{n, m}:=k_{\infty}^{H_{n} \rtimes \Gamma_{n}}=k\left(\mu_{p^{m+1}}, \alpha^{p^{-n}}\right), 0 \leq n, m \leq \infty$, for the intermediate fields of $k_{\infty} / k$. Analogous to the module $X_{n r}$ we have the $\Lambda(\Gamma)$ modules $X_{n r}\left(k_{n, \infty}\right.$ for every field $k_{n, \infty}=k_{c y c l}\left(\alpha^{p^{-n}}\right)$. By class field theory there are canonical isomorphisms of $\Lambda(\Gamma)$ - and $\Lambda(G)$-modules respectively:

$$
\begin{aligned}
X_{n r}\left(k_{n, \infty}\right) & \cong \overleftarrow{\lim }_{m} C l\left(k_{n, m}\right)(p) \\
X_{n r} & \cong \lim _{n, m} C l\left(k_{n, m}\right)(p) \\
& \cong \lim _{n} X_{n r}\left(k_{n, \infty}\right) .
\end{aligned}
$$

Here $C l(K)(p)$ denotes the $p$-part of the ideal class group of a (possibly infinite) extension $K$ of $\mathbb{Q}$. Y. Ochi has proven that $X_{n r}$ is a finitely generated torsion $\Lambda(G)$-module.

Theorem 8.2. Let $\mu_{p} \subseteq k$ and $k_{\infty}=k\left(A_{p \infty}\right)$ as above and suppose that there is only one prime $\mathfrak{p}$ of $k$ above $p$ and that $\mathfrak{p}$ is totally ramified in $k_{\infty} / k$. If $k_{\infty} / k$ is unramified outside $\mathfrak{p}$, then there are canonical isomorphisms

$$
\left(X_{n r}\right)_{H_{n}} \cong X_{n r}\left(k_{n, \infty}\right)
$$

and

$$
\left(X_{n r}\right)_{G_{n}} \cong C l\left(k_{n, n}\right)(p) .
$$

If, in addition, the $\mu$-invariant $\mu\left(X_{n r}\left(k_{c y c l}\right)\right)=0$ is zero, then the following holds:

(i) The $\mu$-invariant is zero for any intermediate field $\mu\left(X_{n r}\left(k_{n, \infty}\right)\right)=0$ and $X_{n r}$ is a finitely generated $\Lambda(H)$-module. In particular, the image of $X_{n r}$ in the quotient category is a (possibly zero) completely faithful, hence cyclic object.

(ii) There is an injective homomorphism of $\Lambda(G)$-modules

$$
C l\left(k_{\infty}\right)(p)^{\vee} \rightarrow \mathrm{E}^{1}\left(X_{n r}\right)
$$

with pseudo-null cokernel, i.e. the Pontryagin dual of the direct limit of the p-ideal class groups is pseudo-isomorphic to the Iwasawa-adjoint of the 
inverse limit. In particular, if $X_{n r}$ is pseudo-null, then the ideal class group $C l\left(k_{\infty}\right)(p)=0$ vanishes.

(iii) There exists a constant $c \geq 0$ independent of $n$ such that

$$
\lambda\left(X_{n r}\left(k_{n, \infty}\right)\right)=\operatorname{rk}_{\Lambda(H)}\left(X_{n r}\right) p^{n}+c
$$

holds for all sufficiently large $n$.

We should mention that until now no single example of $X_{n r}$ not being pseudonull is known. On the contrary, at least for the first irregular prime $p=37$, W.G. McCallum and R.T. Sharifi show in [22] that for $k=\mathbb{Q}\left(\mu_{37}\right)$ and $k_{\infty}=$ $k\left(\mu_{37^{\infty}}, 37^{37^{-\infty}}\right)$ the Greenberg conjecture holds, i.e. $X_{n r}\left(k_{\infty}\right)$ is a pseudo-null $\Lambda(G)$-module. Presumably, this is true for all primes, because otherwise the set of irregular primes would be divided into two classes corresponding to whether the Greenberg conjecture for the above extension holds or not.

Before we can prove the theorem we need some preparation. For a discrete $G_{k^{-}}$ module $N$ we set $N^{*}:=\operatorname{Hom}_{\mathbb{Z}_{p}}\left(N, \mathbb{Q}_{p} / \mathbb{Z}_{p}\right)$ where this module is endowed with the discrete topology.

Lemma 8.3. For every $\Lambda$-module $M$ there is a natural exact sequence

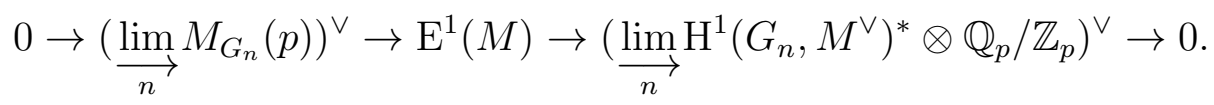

In particular, if $M$ is pseudo-null, then the direct limit $\underset{n}{\lim } M_{G_{n}}(p)=0$ vanishes.

Proof. This is just the Pontryagin dual of [25, (5.4.13)(i)] for $r=1$.

Proposition 8.4. Let $M$ be a $\Lambda(G)$-module which is finitely generated over $\Lambda(H)$.

(i) Assume that $M_{G_{n}}$ is finite. Then $\left(M_{H_{n}}\right)^{\Gamma_{n}}$ is finite, too.

(ii) Assume that $M$ has no non-zero pseudo-null submodule. Then it holds that

$$
\mathrm{H}^{1}\left(G_{n}, M^{\vee}\right) \cong\left(\left(M_{H_{n}}\right)^{\Gamma_{n}}\right)^{\vee} .
$$

If, in addition, $M_{G_{n}}$ is finite for all $n$, then we obtain an isomorphism

$$
\left.\underset{n}{\left(\lim _{n}\right.} M_{G_{n}}\right)^{\vee} \cong \mathrm{E}^{1}(M) \text {. }
$$

(iii) Assume again that $M_{G_{n}}$ is finite for all $n$. Then there is a canonical injection

$$
\left.\underset{n}{\left(\lim _{n}\right.} M_{G_{n}}\right)^{\vee} \rightarrow E^{1}(M)
$$

with pseudo-null cokernel.

Proof. The first item follows from the exact sequence

$$
0 \rightarrow\left(M_{H_{n}}\right)^{\Gamma_{n}} \rightarrow M_{H_{n}} \rightarrow M_{H_{n}} \rightarrow M_{G_{n}} \rightarrow 0
$$

noting that $M_{H_{n}}$ is a finitely generated $\mathbb{Z}_{p}$-module. Now assume that $M$ has no non-zero pseudo-null submodule, i.e. $M$ is a torsion-free $\Lambda(H)$-module (cf. [26, lem 6.4 ]). Thus $\left(M^{H_{n}}\right)_{\Gamma_{n}}=0$ and the first part of (ii) follows from the Hochschild-Serre long exact sequence 


$$
0 \rightarrow\left(\left(M_{H_{n}}\right)^{\Gamma_{n}}\right)^{\vee} \rightarrow \mathrm{H}^{1}\left(G_{n}, M^{\vee}\right) \rightarrow\left(\left(M^{H_{n}}\right)_{\Gamma_{n}}\right)^{\vee}=0
$$

while the second part is a consequence of the first part, (i) and lemma 8.3. To prove (iii) we denote by $M_{0}$ the maximal pseudo-null submodule of $M$ and we set $N:=M / M_{0}$. The exact sequence

$$
0 \rightarrow M_{0} \rightarrow M \rightarrow N \rightarrow 0
$$

induces via the long exact sequence of $\mathrm{E}^{i}$ the exact sequence

$$
\mathrm{E}^{0}\left(M_{0}\right)=0 \rightarrow \mathrm{E}^{1}(N) \rightarrow \mathrm{E}^{1}(M) \rightarrow 0=E^{1}\left(M_{0}\right)
$$

using the fact that $\mathrm{E}^{0}\left(M_{0}\right)=\mathrm{E}^{1}\left(M_{0}\right)=0$ vanishes for pseudo-null modules. On the other hand we obtain taking co-invariants

$$
0=N^{H_{n}} \rightarrow\left(M_{0}\right)_{H_{n}} \rightarrow M_{H_{n}} \rightarrow N_{H_{n}} \rightarrow 0
$$

and

$$
\left(N_{H_{n}}\right)^{\Gamma_{n}} \rightarrow\left(M_{0}\right)_{G_{n}} \rightarrow(M)_{G_{n}} \rightarrow(N)_{G_{n}} \rightarrow 0 .
$$

First note that $(N)_{G_{n}}$ and hence $\left(N_{H_{n}}\right)^{\Gamma_{n}}$ are finite for all $n$ by (i). Thus also $\left(M_{0}\right)_{G_{n}}$ is finite for all $n$. So, by lemma 8.3, $\underset{n}{\lim } M_{G_{n}} \cong \underset{n}{\lim } N_{G_{n}}$ and the result follows from the above isomorphism 8.4 and (ii).

Proof. (of the theorem) The first statement is proved by the same arguments as for $\mathbb{Z}_{p}$-extensions, see e.g. [25, lem. 11.1.5]. Now assume that the $\mu$-invariant vanishes. Nakayama's lemma tells that $X_{n r}$ is a finitely generated $\Lambda(H)$-module, i.e. $X\left(k_{n, \infty}\right)=\left(X_{n r}\right)_{H_{n}}$ a finitely generated $\mathbb{Z}_{p}$-module and thus $\mu\left(X\left(k_{n, \infty}\right)\right)=0$. Item (ii) follows from the above proposition while (iv) is a consequence of the next lemma.

Lemma 8.5. Let $M$ be a $\Lambda(G)$-module which is finitely generated as $\Lambda(H)$ module of rank $\operatorname{rk}_{\Lambda(H)} M=d$. Then there is a constant $c \geq 0$ such that

$$
\mathrm{rk}_{\mathbb{Z}_{p}} M_{H_{n}}=d p^{n}+c
$$

for all sufficiently large $n$.

Proof. Since $M$ is finitely generated as $\Lambda:=\Lambda(H) \cong \mathbb{Z}_{p}[[X]]$-module, by the (commutative) structure theory we obtain an exact sequence

$$
0 \rightarrow M / M_{0} \rightarrow \Lambda(H)^{d} \oplus \bigoplus_{i=0}^{s} \Lambda / \Lambda F_{i} \oplus \bigoplus_{j=0}^{r} \Lambda / \Lambda p^{n_{j}} \rightarrow E \rightarrow 0,
$$

where $M_{0}$ denotes the maximal finite submodule of $M, E$ is finite, the $n_{j} \geq 1$, $0 \leq r$, are natural numbers and the $F_{i} \epsilon \Lambda, 0 \leq i \leq s$, are Weierstrass polynomials. Taking $H_{n}$-coinvariants and tensoring with $\mathbb{Q}_{p}$ one sees immediately that

$$
\operatorname{rk}_{\mathbb{Z}_{p}} M_{H_{n}}=\operatorname{rk}_{\mathbb{Z}_{p}} \mathbb{Z}_{p}\left[H / H_{n}\right]^{d}+\sum_{i=0}^{s} \operatorname{rk}_{\mathbb{Z}_{p}}\left(\Lambda / \Lambda F_{i}\right)_{H_{n}}
$$

holds. Since the second summand is bounded by $\sum_{i=0}^{s} \operatorname{rk}_{\mathbb{Z}_{p}} \Lambda / \Lambda F_{i}<\infty$, it becomes stationary for large $n$ and the result follows. 
The asymptotic behaviour of the $\lambda$-invariant suggest the following asymptotic growth of the order of the $p$-ideal class group. If $e_{n}$ denotes the precise exponent of $\# C l\left(k_{n, n}\right)(p)=p^{e_{n}}$ with respect to $p$, then I guess that

$$
e_{n}=\left(\operatorname{rk}_{\Lambda(H)} X_{n r} \cdot n+O(1)\right) p^{n}
$$

holds.

We finish this paper with a corollary on certain units which are linked to the module $X_{n r}$ via Kummer theory. To this end, we denote by $\mathcal{O}_{K}$ the ring of integers of a number field $K$ and its units by $E(K):=\mathcal{O}_{K}^{\times}$. We set $E\left(k_{\infty}\right)=\underline{\lim _{\longrightarrow}} E\left(k^{\prime}\right)$ where the direct limit runs through all finite subextensions $k\left|k^{\prime}\right| k_{\infty}$. Then

$$
\mathcal{E}\left(k_{\infty}\right):=\left(E\left(k_{\infty}\right) \otimes_{\mathbb{Z}} \mathbb{Q}_{p} / \mathbb{Z}_{p}\right)^{\vee}
$$

is a compact $\Lambda(G)$-module which fits into the following exact and commutative diagram (see [29, prop. 4.15])

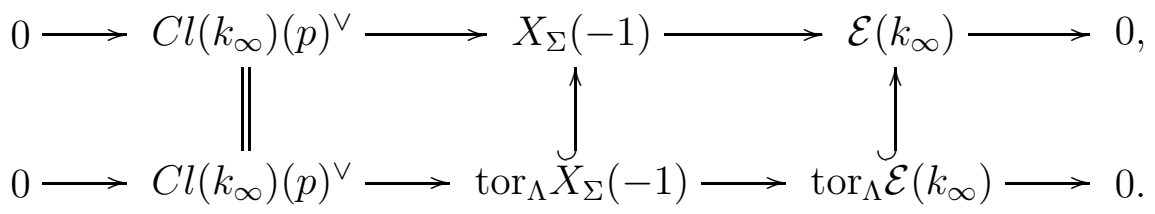

Here, $\Sigma:=S_{p} \cup S_{\infty}$ and $X_{\Sigma}(-1)=\mathrm{H}^{1}\left(G_{\Sigma}\left(k_{\infty}\right), \mu_{p^{\infty}}\right)^{\vee}$ is the first negative Tate twist of the Galois group $G_{\Sigma}\left(k_{\infty}\right)^{a b}(p)$ of the maximal outside $\Sigma$ unramified abelian $p$-extension $k_{\Sigma}^{a b}(p)$ over $k_{\infty}$. With other words, $G_{\Sigma}\left(k_{\infty}\right)$ is the Galois group of the maximal outside $\Sigma$ unramified extension $k_{\Sigma}$ of $k$ over $k_{\infty}$ and $G_{\Sigma}\left(k_{\infty}\right)^{a b}(p)$ its maximal abelian pro- $p$ quotient. It follows from [29, thm 3.1.5, cor. 3.1.6], that $\operatorname{tor}_{\Lambda} X_{\Sigma}(-1)$ is pseudo-isomorphic (in the quotient category) to $\mathrm{E}^{1}\left(X_{n r}\right)$. Finally, as a consequence of the above theorem we obtain

Corollary 8.6. Under the assumptions of the theorem the torsion submodule $\operatorname{tor}_{\Lambda} \mathcal{E}\left(k_{\infty}\right)$ of $\mathcal{E}\left(k_{\infty}\right)$ is pseudo-null.

\section{REFERENCES}

[1] G.Q. Abbasi, S. Kobayashi, H. Marubayashi, and A. Ueda, Non commutative unique factorisation rings, Commun. Algebra 19 (1991), no. 1, 167-198.

[2] B. J. Birch, Cyclotomic fields and Kummer extensions, Algebraic Number Theory (Proc. Instructional Conf., Brighton,1965), Thompson, Washington, D.C., 1967, pp. 85-93.

[3] N. Bourbaki, Éléments de mathématique. Fasc. XXXI. Algèbre commutative. Chapitre \%: Diviseurs, Hermann, Paris, 1965.

[4] _ Éléments de mathématique. algèbre commutative. chapitre 8. dimension. chapitre 9. anneaux locaux noethériens complets, Masson, Paris, 1983.

[5] A. Brumer, Pseudocompact algebras, profinite groups and class formations, J. of Algebra 4 (1966), 442-470.

[6] M. Chamarie, Anneaux de Krull non commutatifs, J. Algebra 72 (1981), no. 1, 210-222.

[7] _ Modules sur les anneaux de Krull non commutatifs., Sém. d'Algèbre P. Dubreil et M.-P. Malliavin 1982, LNM, vol. 1029, Springer, 1983, pp. 283-310. 
[8] A. W. Chatters, Noncommutative unique factorization domains, Math. Proc. Cambridge Philos. Soc. 95 (1984), no. 1, 49-54.

[9] A. W. Chatters and D. A. Jordan, Noncommutative unique factorisation rings, J. London Math. Soc. (2) 33 (1986), no. 1, 22-32.

[10] J. Coates, R. Sujatha, and P. Schneider, Modules over Iwasawa algebras, preprint (2001).

[11] P. M. Cohn, Skew fields, Cambridge University Press, Cambridge, 1995, Theory of general division rings.

[12] D. Burns and C. Greither, Equivariant Weierstrass Preparation and values of L-functions at negative integers, preprint (2002).

[13] J.D. Dixon, M.P.F. du Sautoy, A. Mann, and D. Segal, Analytic pro-p groups, 1st ed., London Mathematical Society Lecture Note, vol. 157, Cambridge University Press, 1991.

[14] K.R. Goodearl and R.B. Warfield, An Introduction to Noncommutative Noetherian Rings, LMS Student texts, vol. 16, Cambridge University Press, 1989.

[15] Y. Hachimori and O. Venjakob, Completely faithful selmer groups over kummer extensions, preprint (2002).

[16] Michael Harris, The annihilators of p-adic induced modules, J. Algebra 67 (1980), no. 1, $68-71$.

[17] S. Howson, Euler Characteristics as Invariants of Iwasawa Modules, preprint (2000).

[18] _ Structure of Central Torsion Iwasawa Modules, preprint (20001).

[19] M. Lazard, Groupes analytiques p-adiques, Publ. Math. I.H.E.S. 26 (1965), 389-603.

[20] H. Li and F. van Oystaeyen, Zariskian filtrations, Kluwer Academic Publishers, Dordrecht, 1996.

[21] W.G. McCallum, Greenberg's conjecture and units in multiple $\mathbb{Z}_{p}$-extensions, preprint (1999).

[22] W.G. McCallum and R.T. Sharifi, Greenberg's conjecture in the nonabelian case, preprint (2002).

[23] J. C. McConnell and J. C. Robson, Noncommutative Noetherian rings, John Wiley \& Sons Ltd., Chichester, 1987, With the cooperation of L. W. Small, A Wiley-Interscience Publication.

[24] J. Nekovar, Selmer complexes, preprint (2001).

[25] J. Neukirch, A. Schmidt, and K. Wingberg, Cohomology of number fields, Grundlehren der mathematischen Wissenschaften, vol. 323, Springer, 2000.

[26] Y. Ochi and O. Venjakob, On the structure of Selmer groups over p-adic Lie extensions, to appear in the Journal of Algebraic Geometry.

[27] J. C. Robson, Cyclic and faithful objects in quotient categories with applications to Noetherian simple or Asano rings, Noncommutative ring theory (Internat. Conf., Kent State Univ., Kent, Ohio, 1975), Springer, Berlin, 1976, pp. 151-172. Lecture Notes in Math., Vol. 545.

[28] O. Venjakob, On the structure theory of the Iwasawa algebra of a p-adic Lie group, to appear in Jour. Eur. Math. Soc.

[29] _ Iwasawa Theory of p-adic Lie Extensions, preprint (2001).

Universität Heidelberg, Mathematisches Institut, Im Neuenheimer Feld 288, 69120 Heidelberg, Germany.

E-mail address: otmar@mathi.uni-heidelberg.de

URL: http://www.mathi. uni-heidelberg.de/〜otmar/ 